\title{
Leukemia-intrinsic determinants of CAR-T response revealed by in vivo genome-wide CRISPR screening
}

\author{
Azucena Ramos, ${ }^{1,2 \dagger}$, Catherine E. Koch ${ }^{1,2 \dagger}$, Yunpeng Liu, ${ }^{1,2 \dagger}$ Riley D. Hellinger, ${ }^{1}$ Taeyoon \\ Kyung, ${ }^{1,3}$ Rebecca C Larson, ${ }^{8,9}$ Julia Fröse, ${ }^{1}$ Daniel Goulet, ${ }^{1}$ John G. Doench, ${ }^{3}$ Aviv \\ Regev, ${ }^{2,4,5,6,7}$ Marcela V. Maus, ${ }^{5,8,9,10}$ Michael E. Birnbaum, ${ }^{1,3,8}$ Michael T. Hemann ${ }^{1,2,4 *}$
}

${ }^{1}$ Koch Institute for Integrative Cancer Research, Massachusetts Institute of Technology, Cambridge, MA, USA

${ }^{2}$ Department of Biology, Massachusetts Institute of Technology, Cambridge, MA, USA

${ }^{3}$ Department of Biological Engineering, Massachusetts Institute of Technology, Cambridge, MA, USA.

${ }^{4}$ Ludwig Center for Cancer Research at MIT, Boston, MA, USA.

${ }^{5}$ Broad Institute of Harvard and Massachusetts Institute of Technology, Cambridge, Massachusetts 02142, USA

${ }^{6}$ Klarman Cell Observatory, Broad Institute of MIT and Harvard, Cambridge, MA, USA.

${ }^{7}$ Howard Hughes Medical Institute, Chevy Chase, MD, USA.

${ }^{8}$ Cellular Immunotherapy Program, Cancer Center, Department of Medicine, Massachusetts General Hospital, Boston, MA, USA.

9Immunology Program, Harvard Medical School, Boston, MA, USA.

${ }^{10}$ Ragon Institute of MIT, MGH, and Harvard, Cambridge, MA, USA.

$\dagger$ These authors contributed equally to this work

*Correspondence: hemann@mit.edu 


\section{Main Text}

\section{Abstract}

CAR-T cells are a promising new treatment for B cell malignancies. However, the majority of patients inevitably go on to experience disease relapse through largely unknown means. To investigate leukemia-intrinsic CAR-T resistance mechanisms, we performed genome-wide CRISPR-Cas9 loss-of-function screening in an immunocompetent murine model of $B$ cell acute lymphoblastic leukemia (B-ALL). We identified IFNy/JAK/STAT signaling and components of the antigen processing and presentation pathway as key mediators of resistance to anti-CD19 CAR-T therapy in vivo, but not in vitro. Transcriptional characterization of this model demonstrated an upregulation of these pathways in CAR-T treated relapsed tumors, and examination of data from CAR-T treated B-ALL patients revealed an association between poor outcomes and increased expression of JAK/STAT/MHC-I in leukemia cells. Together, our data identify an unexpected mechanism of resistance to immunotherapy, in which tumor cell interaction with IFNy-secreting CAR-T cells in vivo activates the expression of an $\mathrm{MHC}-\mathrm{I}$ inhibitory T cell program.

\section{Introduction}

Immunotherapies have emerged as crucial components of cancer treatment and are quickly becoming the standard of care in a broad range of malignancies. ${ }^{1}$ One of the most promising immunotherapy agents today is the adoptive cell transfer (ACT) of autologous T

22 lymphocytes engineered to express chimeric antigen receptors (CARs). ${ }^{2,3}$ Functionally, CARs redirect the cytotoxicity of immune cells towards a patient's tumor. Groundbreaking trials in

24 relapsed B cell malignancies demonstrated extraordinary initial efficacy, with upwards of $90 \%$ of patients experiencing complete responses (CR). ${ }^{4-8}$ Despite these impressive results, clinical data from CAR-T treated patients suggest that relapse is likely to be a significant and ongoing problem in this treatment modality. Recent studies have shown that while the median overall survival after CAR-T therapy is on the order of 12 to 20 months, upwards of $60 \%$ of patients will experience disease recurrence. ${ }^{5,8-13}$ Further, up to $20 \%$ of B-ALL patients never achieve remission and applications to other $\mathrm{CD} 19^{+} \mathrm{B}$-cell malignancies such as chronic lymphocytic leukemia $(C L L)$ and diffuse large B-cell lymphoma (DLBCL) have significantly lower CR

32 rates. $5,8,11,14$ Thus, gaining a more complete understanding of the factors governing response in CAR-T therapy may ultimately lead to improvements in CAR-T cell design or combination therapies that maintain remission and improve patient outcomes. 


\section{Ramos, Koch, and Liu et al: Confidential}

To date, a limited number of CAR-T resistance mechanisms have been reported in the literature. ${ }^{15}$ Treatment failure can be associated with loss of the infused CAR-T cell product, particularly in patients who never achieve remission. ${ }^{16,17}$ This has led to the hypothesis that CAR-T cell dysfunction is a central resistance mechanism in this treatment modality. Accordingly, significant efforts to characterize $T$ cell dysfunction have been undertaken, and

40 factors such as the quality of harvested T-cells and variations in production and manufacturing have all been implicated. Leukemia-intrinsic changes have also been described, with target

42 antigen loss arguably garnering the most attention. ${ }^{18,19}$ Overall, relapse with CD19- disease has been shown to occur in up to one in four patients experiencing treatment failure. ${ }^{20,21}$ In the case

44 of anti-CD19 CAR-T therapy, tumor cell loss of CD19 has been found to occur via various mechanisms, including mutations in the CD19 locus, alternative splicing of CD19 mRNA, and

46 through lineage switching. ${ }^{15}$ Decidedly less is known about the underlying drivers of relapse in CD19 ${ }^{+}$disease. Here, CAR-T cell dysfunction has again been posited as a contributor to

48 treatment failure. However, it is presently unclear to what degree intrinsic CAR-T dysfunction drives relapse in these patients, or whether pre-existing or acquired tumor cell adaptations can function to facilitate or incite CAR-T dysfunction, independent of CD19 status. Interestingly, recent reports support a role for the latter idea. For example, Singh and colleagues showed that impaired death receptor signaling in tumor cells can lead to resistance by inducing CAR-T cell dysfunction. ${ }^{22}$ Gene expression analysis of pre-CAR-T treated patient samples demonstrated that pre-existing tumor cell expression profiles in the death receptor pathway differed between patients achieving CRs and those who never responded to CAR-T therapy. Strikingly, these preexisting expression profiles successfully predicted therapeutic outcomes in an independent patient cohort, suggesting that in some cases, CAR-T resistance may arise from pre-existing tumor cell alterations. These data, along with the paucity of known resistance mechanisms outside of target antigen loss or CAR-T cell dysfunction, highlight the urgent need to carefully examine the role of tumor cell alterations in CAR-T treatment failure.

To systematically investigate potential mechanisms of leukemia-intrinsic resistance to CAR-T cells in an unbiased manner, we performed parallel whole genome in vitro and in vivo CRISPR/Cas9-mediated loss-of-function (LOF) screens for factors involved in response and resistance to anti-CD19 CAR-T cell treatment in a transplantable, immunocompetent mouse model of $B C R-A B L^{+} B$ cell acute lymphoblastic leukemia (B-ALL). ${ }^{23,24}$ We then compared our top in vivo-specific hits with single cell expression analysis data of relapsed B-ALL cells after CAR-T treatment, and with published patient clinical data. The resulting data indicate that the interactions between interferon gamma (IFNY)-secreting CAR-T cells and tumor cells can 
promote leukemia-specific alterations that can fuel relapse. These findings suggest an

"adaptive" resistance mechanism to cell-based immunotherapy and point to new possibilities for enhancing CAR-T cell efficacy without modifying existing CAR-T cell products.

\section{Results}

74 A fully immunocompetent mouse model of BCR-ABL1 ${ }^{+}$B-ALL enables parallel in vivo and in vitro genome-wide screens for CAR-T resistance

Using an established mouse model of $B C R-A B L 1^{+} B-A L L$ with a high engraftment rate in immunocompetent, syngeneic recipient mice, we engineered Cas 9 expressing cells

78 (Supplemental Figure 1a) with high cutting efficiencies (Supplemental Figure 1b) to allow for unbiased genome-wide screens. ${ }^{23-27}$ To determine if an in vivo screen for CAR-T resistance

80 using immunocompetent hosts was tractable, we examined in vivo growth in both wildtype (WT) and $\mathrm{Cas}^{+}$cells, reasoning that if Cas 9 expression induced any immunogenic barrier, this would manifest as delayed growth kinetics over time. A luciferase ${ }^{+}$Cas $^{+}$clone $(20.12)$, growth matched its WT parental line in vitro was transplanted into non-irradiated immunocompetent male C57BL/6J (B6) mice. Compared to the WT parental line, no significant growth delays in Cas $9^{+}$cells could be detected in vivo, in any hematopoietic organ assayed (Supplemental Figure 1c). Parallel experiments were also performed in non-irradiated male B6 mice and immunocompromised NOD-SCID/IL2 $\mathrm{Rg}^{-/-}$(NSG) mice. If Cas9 was immunogenic in recipient mice, immunosuppressed NSG mice transplanted with Cas ${ }^{+}$cells would have succumbed to disease faster than immunocompetent B6 mice transplanted with the same cells. However, no differences in disease latency were observed in repeated experiments (Supplemental Figure 1d).

We then examined the ability of murine CAR-T cells to suppress tumor cell growth in vivo. As patients treated with CAR-T therapy undergo lymphodepletion with cyclophosphamide or irradiation prior to therapy, ${ }^{28-30}$ we first subjected recipient mice to an irradiation-based lymphodepletion protocol prior to tumor transplantation and subsequent CAR-T treatment. To calibrate the dose of CAR-T cells, we performed a series of in vivo dosing experiments utilizing CD28-based $2^{\text {nd }}$ generation murine CARs targeting murine CD19 (mCD19). Previous groups utilizing a similar construct successfully suppressed disease with CAR-T dose ranges in the millions of cells $\left(5 \times 10^{6}\right.$ to $\left.2 \times 10^{7}\right)$ per animal. ${ }^{28,29,31}$ In our model, doses of $7 \times 10^{6}$ to $1 \times 10^{7}$ CAR-T

100 cells per animal, administered two days after the transplantation of $0.6 \times 10^{6} \mathrm{~B}-\mathrm{ALL}$ cells achieved significant dose-dependent life extension (Figure 1a). To simultaneously monitor

102 disease suppression in real time, we used leukemia cells engineered to express firefly 
luciferase. ${ }^{26}$ Bioluminescence imaging completed at multiple time points after ACT

104 demonstrated that anti-mCD19 CAR-T cells could significantly suppress disease over time (Figure 1b-f).

To ensure CAR-T functionality, we conducted concurrent matched in vitro cytotoxicity assays for each independent experiment and measured CAR-T expansion and IFNY (a cytokine released by $T$ cells in proinflammatory conditions) in the resulting culture supernatant. Interestingly, murine B-ALL cell numbers could be significantly reduced but never fully

110 eliminated in vitro, even at very high effector to target (E:T) ratios (Figure $1 \mathrm{~g}$ ). CAR-T cells also induced a rapid and dramatic loss of the MCD19 target epitope on the surface of B-ALL cells

112 (Figure $1 \mathrm{~h}$ ), a phenotype that our results suggest is target antigen-independent and not a unique feature of our murine B-ALL model (Supplementary Figure 1e). Importantly, while

114 antigen loss was a primary phenotype in our B-ALL cells, our cytotoxicity assays still resulted in activated CAR-T cells that expanded (Figure 1i) and released significant IFNy after being cocultured with cells expressing their target antigen (Figure $1 \mathrm{j}$ ).

Our group has previously shown that this mouse model of B-ALL is highly amenable to 118 in vivo LOF screens. ${ }^{25,26}$ Thus, we next sought to determine the CAR-T cell dose that would adequately suppress disease in a pooled in vivo screening setting. ${ }^{25,26}$ Irradiated B6 mice were

120 transplanted with B-ALL cells (clone 20.12), treated with varying amounts of CAR-T cell doses, and monitored recipient mice using bioluminescence imaging. Animals were sacrificed at peak

122 disease suppression which occurred on or before day 3 after adoptive cell transfer (ACT) of CAR T cells (Supplementary Figure 1f). Total disease burden, target tumor antigen expression,

124 and CAR-T expansion were assayed in various hematopoietic organs. We aimed for an $80-90 \%$ disease suppression rate, as this significant but incomplete level of tumor cell reduction would

126 allow for identification of alterations that could sensitize tumor cells to therapy. For the bone marrow compartment, this was accomplished using $1.5 \times 10^{7}$ CAR-T cells, while splenic and

128 peripheral blood compartments only required a dose of $1 \times 10^{7}$ CAR-T cells (Figure 1k). Relapsed disease harvested from CAR-T treated animals consistently showed a striking dose-dependent

130 loss of mCD19 surface expression along with significant CAR-T engraftment in all organs examined (Figure 1I-m), a consistent phenotype observed across all in vivo experiments. Given

132 that we also observe significant antigen loss and concomitant CAR-T cell persistence in relapsed animals (Supplemental Figure $1 \mathrm{~g}$ ), we reasoned that this phenotype was due to

134 ongoing CAR-T surveillance. Indeed, when mCD19 negative leukemia cells were harvested from relapsed mice and cultured in vitro, mCD19 expression was restored as CAR-T cells were 136 depleted from culture (Supplementary figure $1 \mathrm{~h}-\mathrm{i}$ ). Notably, human data has demonstrated that 


\section{Ramos, Koch, and Liu et al: Confidential}

as many as half of all patients relapse with antigen negative disease. Thus, our system may model the antigen loss and leukemia relapse seen following CAR-T therapy in a large proportion of patients. .,9,32 $^{2}$

\section{Unbiased CRISPR/Cas9-mediated screen identifies genes and pathways involved in in}

\section{vivo resistance to anti-CD19 CAR-T therapy}

Having established a mouse model of anti-CD19 CAR-T treatment response, we next

144 sought to develop a CRISPR/Cas9 library that would enable genome-wide in vivo screens across a broad range of tumor models with varying engraftment rates. We generated a custom

146 single guide (sgRNA) library cloned into an optimized lentiviral backbone (Figure 1n-o). The library is composed of 48 sub-pools, each targeting the protein coding regions of approximately

148430 unique murine genes. Importantly, sub-pools can be used as stand-alone screening libraries, as each contains all requisite controls needed for a screen. Given the high tumor

150 engraftment rate in our B-ALL model, we were able to pool our 48 sub-pools into groups of eight, limiting our entire genome-wide in vivo and in vitro screens to six screens completed over two experiments using our CD28-based $2^{\text {nd }}$ generation murine CAR constructs (Figure $1 \mathrm{p}$ ).

To query factors responsible for CAR-T resistance, we targeted 21,958 protein coding genes and subjected cells to either anti-CD19 CAR-T therapy or control CAR-T cells (targeting anti-hEGFRvIll which is not expressed in mice or on our B-ALL model) in vitro or in vivo. The in vivo arm of our screen followed the layout of our optimized dose finding experiments. We observed that relapsed CAR-T treated mice harbored both CD19-positive and -negative disease, as well as persistent CAR-T cell populations in both the spleen and bone marrow (Supplementary Figure $2 a$ and b, respectively). Concurrent with this in vivo screen, we performed parallel in vitro screens at two effector to target (E:T) ratios. (Figure 1q). We then used high-throughput sequencing to quantify sgRNA representation in B-ALL cells harvested in each experimental arm. Importantly, we were able to confirm that we achieved extensive coverage of every sgRNA library pool screened (Supplementary Figure 2c-d). To confirm that our screen was able to effectively interrogate gene function while remaining unaffected by possible artifacts from Cas9-mediated DNA breakage, we quantified the enrichment and depletion of sgRNAs in the control treatment group compared to pre-screen input samples. Indeed, sgRNAs targeting known essential genes significantly depleted in the control treatment groups, whereas those targeting intergenic regions as well as non-targeting sgRNAs did not (Supplementary Figure 2e). 


\section{Ramos, Koch, and Liu et al: Confidential}

As expected, samples treated with anti-CD19 CAR-T therapy demonstrated patterns of sgRNA enrichment and depletion that were largely distinct from control treated groups

172 (Supplementary Figure 2f). To assess the genetic dependencies involved in the response of BALL cells to anti-CD19 CAR-T treatment, we compared the anti-CD19 CAR-T and control

174 treatment arms of the screen across each of the 6 pools (Figure 2a). These results were then aggregated to generate a genome-wide perturbation landscape of CAR-T treatment response

176 (Figure 2b-c). Within such a landscape, sgRNAs depleting in response to CAR-T treatment target genes that putatively mediate resistance to CAR-T therapy, whereas those that enrich

178 target genes that likely sensitize B-ALL cells to CAR-T therapy. Two central features support the biological relevance of our findings; first, sgRNAs targeting the Cd19 locus are the top overall

180 enrichers (Figure $2 \mathrm{~b}-\mathrm{e}$ ) and second, the magnitude of enrichment and depletion appears to be positively associated with the dosage of anti-CD19 CAR-T cells administered (i.e. the selective

182 pressure experienced by target cells) (Figure 2b, left and middle panels and Figure 2c). Importantly, a comparison of top hits in the in vivo and in vitro arms of our screen (Figure 2f)

184 showed little overlap between hits from these two arms, suggesting that a co-culture screen modality is incapable of capturing key in vivo mechanisms of CAR-T treatment resistance. In

186 fact, certain sgRNAs show the opposite behavior in each setting. For example, several top enrichers in the in vitro arms of the screen, such as sgRNAs targeting the IFNy signaling 188 pathways members Janus kinase 2 (Jak2), signal transducer and activator of transcription proteins (Stat1), and interferon gamma receptor 1 (Ifngr1), are found to be depleted in the in 190 vivo arms (Figure 2d, e).

We next sought to determine whether pathways independent of target molecule (CD19)

192 expression were involved in resistance to CAR-T therapy. Using gene set enrichment analysis (GSEA), we found that several signaling and metabolic processes within the MSigDB hallmark

194 gene sets, such as the JAK/STAT/IL-6 signaling axis, PI3K/AKT pathway, and oxidative phosphorylation (OXPHOS) were enriched among genes that potentially mediate resistance

196 (Figure 2g, depleters, above dotted line). In contrast, some of these pathways are enriched among genes that potentially sensitize cells to therapy in vitro (Figure $2 \mathrm{~g}$, below dotted line).

198 Additionally, enrichment analysis of KEGG pathway terms (Figure $2 \mathrm{~h}$ ) showed that top depleters in the in vivo arms of our screen are enriched for processes such as tumor necrosis factor (TNF)

200 signaling, immune checkpoint signaling, and immune response against infectious diseases. Interestingly, the interferon gamma receptor 1 (IFNGR1) along with JAK/STAT/IL-6 signaling

202 pathway components, and the murine histocompatibility 2, T region locus 23 (H2-T23, also known as $\mathrm{Qa}-1^{\mathrm{b}}$ ) are components of multiple enriched pathways, suggesting that these genes 


\section{Ramos, Koch, and Liu et al: Confidential}

204 may be at the center of cellular response to CAR-T therapy in vivo. Indeed, sgRNAs against these genes are among the top in vivo-specific depleters (Figure $2 \mathrm{~d}, \mathrm{e}$ ).

\section{The IFNy pathway promotes resistance to CAR-T therapy in vivo}

To further validate our findings, and particularly, hits in the JAK/STAT/IFNY pathway, we conducted parallel in vivo and in vitro competition experiments (Figure 3a-c and Supplementary

210 Figure 3a). Here, we used a new, independently generated Cas9-expressing cell line (RH62) from our parental B-ALL model with high cutting efficiency and matched in vitro growth kinetics

212 as our screened clone (Supplemental Figure 3b, c). We focused on genes that when lost, significantly depleted in the in vivo arms of our screen, as they could represent drug targets that,

214 when inhibited, might potentiate the effects of CAR-T therapy. Using competition assays that compare the relative growth of mixtures of isogenic knock out or control B-ALL cells both in vitro

216 and in vivo, we found that the proportion of cells null for Ifngr1, Jak2, or Stat1 significantly depleted in both the marrow and spleens of mice treated with anti-CD19 CAR-T therapy (Figure

$2183 b$, c showing log-scale depletion). Notably, no growth differences were noted in mice transplanted with identical cell mixtures but treated with control CAR-T cells, indicating that this

220 phenotype is not driven by fitness defects imparted on cells that have lost these genes. Interestingly, parallel in vitro experiments again demonstrated the opposite result, with Ifngr1,

222 Jak2, or Stat1 knock out cells significantly enriching in the context of anti-CD19 CAR-T therapy (Supplementary Figure 3a). Ultimately, similar to our screen findings, these data demonstrate

224 that loss of Ifngr1, Jak2, or Stat1 sensitizes B-ALL cells to CAR-T therapy in vivo while promoting resistance in vitro.

To further validate our in vivo results, we generated pure populations of leukemic cells deficient in Ifngr1, Jak2, or Stat1 (Supplementary Figure 3d). Consistent with our previous data,

228 mice transplanted with these cells demonstrated an enhanced response to CAR-T therapy, resulting in significantly increased survival (Figure 3d and Supplementary Figure 3e) and even

230 curative outcomes. Interestingly, a parallel experiment conducted in NSG animals demonstrated that tumors deficient in Ifngr1 remain highly sensitized to CAR-T therapy (Figure 3e), indicating

232 that this sensitization phenotype is largely dependent on interactions between tumor and CAR-T cells. Thus, tumor cells can directly co-opt IFNy signaling in vivo to resist killing by CAR-T cells

234 using a mechanism that is independent of other cellular components of the adaptive immune system. Conversely, CAR-T efficacy in vitro appears to be at least partially dependent on the

236 ability of tumor cells to sense IFNy. Together, these data highlight the significant difference between in vitro and in vivo contexts and demonstrate the importance of investigating tumor cell 


\section{Ramos, Koch, and Liu et al: Confidential}

238 response to CAR-T therapy in a physiologically relevant setting. These results also highlight a central role for the JAK/STAT/IFNY pathway in promoting resistance to CAR-T therapy in B-ALL.

\section{Global targeting of JAK/STAT signaling does not enhance CAR-T therapy}

Having established the JAK/STAT/IFNY pathway as a key mediator of response to CART therapy, we next explored whether global blocking of IFNy in vivo could potentiate the effects of CAR-T therapy. To this end, we administered blocking antibodies targeting IFNY the day before ACT, and every 3 days thereafter (Figure 3f). Rather than enhancing antitumor effects,

246 blocking IFNY in the context of anti-mCD19 CAR-T treatment abrogated the anti-tumor effects of CAR-T cells. Here, mice treated with anti-CD19 and anti-IFNy antibody succumb to disease at

248 the same time as mice treated with control CAR-T cells and an isotype control antibody (Figure $3 g$ and Supplementary Figure 3f). Identical results were obtained when animals were co-treated 250 with ruxolitinib and CAR-T therapy in a similar experiment (Figure 3h, i and Supplementary Figure $3 g-m)$. These data are consistent with results from recent studies which show that

252 drugging CAR-T cells with JAK/STAT inhibitors or knocking out Ifng in CAR-T cells significantly impairs their ability to eliminate tumor cells in vitro and in vivo, respectively. ${ }^{31,33}$ Thus, IFNy is

254 indispensable for sustaining CAR-T cell cytotoxicity in vivo, resulting in the lack of a therapeutic window for targeting this cytokine directly to sensitize tumor cells to CAR-T cell killing.

\section{H2-T23 is an in vivo-specific mediator of resistance to CAR-T therapy} and expression patterns that have been shown to be induced by IFNY/JAK/STAT

260 proinflammatory signaling. One of the most promising factors fitting these criteria is the nonclassical class I major histocompatibility complex (MHC-I) gene Histocompatibility 2, T region

262 locus 23 (H2-T23) encoding Qa-1 ${ }^{\mathrm{b}}$, the murine homolog of human leukocyte antigen (HLA)E. ${ }^{34,35}$ HLA-E is the only known ligand of the CD94/NKG2A receptor which is expressed on the

264 surface of natural killer (NK) and $\mathrm{CD}^{+}$T cells. $^{36-40}$ Binding of NKG2A/CD94 to its ligand transmits a signal that inhibits the effector functions of NK and CD8 ${ }^{+}$T cells (Figure $4 a$ ). ${ }^{38-42}$

266 Recently, several groups have reported significant enhancement in the antitumor effects of immune checkpoint blockade (ICB) and cancer vaccines when combined with blockade of the

268 HLA-E-NKG2A axis, and early clinical trials have demonstrated encouraging results in a variety of cancers, including hematologic malignancies. ${ }^{35,41-43}$ To explore the possibility that inhibition of

270 this axis could also potentiate the effects of CAR-T therapy in vivo, we set out to validate $\mathrm{H} 2$ T23, again using competition assays. As observed in the primary screen, loss of H2-T23 in B- 


\section{Ramos, Koch, and Liu et al: Confidential}

272 ALL cells does not change the in vitro response to CAR-T therapy (Supplemental Figure 4a). Remarkably, most animals bearing tumors partially transduced with sgRNAs against $H 2-T 23$

274 relapsed with bone marrow disease that was substantially CD19 positive after CAR-T therapy, a phenotype that was not observed in any other set of CAR T experiments or any other mice

276 receiving the same CAR-T cells in parallel experiments (Figure 4b, c). Interestingly, this phenotype also appears to be limited to the bone marrow, as relapsed disease in the spleen of

278 these mice was predominantly CD19 negative (Supplementary figure 4b, c).

To further examine the consequence of $\mathrm{Qa}-1^{\mathrm{b}}$ loss on CAR-T response, we generated

280 pure populations of Qa-1 ${ }^{\mathrm{b}}$ null B-ALL lines by sorting for bulk sgRNA-bearing cells that could no longer be induced to express Qa- $1^{\text {b }}$ (Supplementary Figure $4 d, e$ ). ${ }^{42}$ Consistent with our

282 previous results, Qa- $1^{\mathrm{b}}$ knockout tumors were sensitized to CAR-T therapy in vivo, leading to significant life extension, including curative outcomes in fully immunocompetent animals (Figure

284 4c). Notably, immunodeficient NSG recipient mice bearing Qa-1 ${ }^{\text {b}}$-deficient tumors showed extended survival in response to CAR-T therapy but, unlike B6 recipients, eventually relapsed,

286 suggesting that full CAR-T efficacy may require other cellular components of the adaptive immune system. Finally, to investigate whether cells deficient in components of the

288 JAK/STAT/IFNY pathway are also functionally null for $\mathrm{Qa}-1^{\mathrm{b}}$, we performed in vitro cytotoxicity experiments. While control cells were fully capable of inducing dose-dependent expression of

290 Qa-1 ${ }^{\text {b }}$ upon exposure to anti-CD19 CAR-T cells, isogenic cell lines deficient in Stat1, Jak2, or Ifngr1 were completely blunted in their ability to express this molecule at all E:T ratios examined

292 (Figure 4d) or when these experiments were repeated using only recombinant IFNY (Supplementary Figure 4f). Together, our data highlight a role for the Qa-1 ${ }^{\mathrm{b}} / \mathrm{NKG} 2 \mathrm{~A} / \mathrm{CD} 94$ axis

294 in promoting resistance to CAR-T therapy via a mechanism that appears to depend on the sensing of IFNy by tumor cells and additional cellular components of the endogenous immune 296 system.

298 A JAK/STAT/IFNy program delineates cells refractory to anti-CD19 CAR-T therapy

To further explore transcriptional programs driving B-ALL CAR-T resistance mechanisms

300 highlighted by our screen and validation experiments, we transcriptionally profiled B-ALL cells after in vivo treatment with CAR-T cells. Principal component analysis of transcription profiles

302 using the most variable genes across samples showed a clear separation of animals treated with anti-CD19 CAR-T cells from those treated with control anti-hEGFRvIII CAR-T cells (Figure

304 5a). Differential expression analysis showed that several genes involved in JAK/STAT signaling and antigen processing and presentation pathways, including Stat1, Irf1, Socs1, and several 


\section{Ramos, Koch, and Liu et al: Confidential}

major histocompatibility complex (MHC) genes, are highly expressed in samples treated with anti-CD19 CAR-T therapy compared to those treated with control CAR-T cells (Figure 5b). Interestingly, sgRNAs targeting these genes are also among the top depleters in the bone marrow or spleen in our in vivo screen, suggesting that these genes are part of an expression program that may contribute to therapeutic resistance. Consistently, GSEA of Hallmark pathways highlighted interferon response, allograft rejection, MYC/2F targets, and IL6/JAK/STAT3 signaling as top pathways enriched in samples treated with anti-CD19 CAR-T cells (Figure 2c).

Like other tumor types, B-ALL often displays a heterogeneous in vivo response to therapy. To dissect the in vivo transcriptional responses to anti-CD19 CAR-T therapy at a finer

316 resolution and pinpoint specific cell populations giving rise to relapse, we performed dropletbased single-cell transcriptome profiling on a total of 124,523 cells harvested from the bone

318 marrow and spleen of mice treated with anti-CD19 or control anti-hEGFRvIII CAR-T cells. Unsupervised clustering revealed 15 distinct cell populations as seen in the 2-dimensional

320 uniform manifold approximation and projection (UMAP) plots in Figure $5 \mathrm{~d}$ (left panel). Cells from different treatment arms and tissues occupy distinct clusters (Figure 4d, right panel and

322 Supplementary Figure 5a). Notably, we did not observe loss of Cd19 transcript expression in any sample (Supplementary Figure 5b, left panel), indicating that loss of CD19 surface expression in this context is likely the result of post-translational regulation. Remarkably, a few clusters $(2,4,5$, and 7$)$ are substantially enriched for cells from anti-CD19 CAR-T treatment groups while being depleted of cells from the control treatment groups (Figure $5 \mathrm{e}$ ). Consistent with our bulk RNAseq data, these clusters are marked by elevated expression of genes in the

328 IFNY signaling and allograft rejection pathways, as well as MYC and E2F target sets (Figure 5f). Importantly, several signature genes in cluster 2 are also among the top depleters in our in vivo screens. Figure $4 \mathrm{~g}$ highlights the magnitude and pervasiveness of the expression of these genes across cell clusters. Additionally, clusters 4, 5, and 7 are enriched for genes involved in

332 oxidative phosphorylation (OXPHOS) and mTORC1 signaling pathways (Figure 5f). Cells in clusters 2 and 4, which contain mostly splenic disease from anti-CD19 CAR-T treated animals,

334 show widespread and high expression of Stat1 and H2-T23 (Figure 5g, h). Intriguingly, transporter associated with antigen processing (Tap1) is also over-expressed in these clusters

336 (Supplementary Figure 5b, right panel). Cluster 7, which is almost entirely made up of cells from anti-CD19 CAR-T treated bone marrow, is marked by a strong G2/M arrest phenotype (Figure $5 f$ 338 and Supplementary Figure 5c) along with high expression of H2-T23 and Stat1 (Figure 5h). Transcription factor (TF) motif analysis of the regulatory regions of genes specifically up- 
340 regulated in clusters 2, 4, 5 and 7 showed enrichment of motifs for critical TFs involved in interferon response, such as ETS family TFs, IRF8 and ELK4, with particularly strong

342 enrichment in clusters 2 and 4 (Supplementary Figure $5 \mathrm{~d}$ ).

Our single-cell expression data, taken together with results from our in vivo screen and

344 validation assays, further support the hypothesis that cross-talk between antigen processing and presentation, and interferon gamma signaling pathways underlie the cellular response to

346 anti-CD19 CAR-T therapy in B-ALL cells. Here, a subset of cells characterized by high expression of genes associated with these pathways may be responsible for therapeutic

348 resistance.

350 High JAK/STAT signaling correlates with CAR-T resistance in humans

Finally, to examine whether JAK/STAT signaling in tumor cells might also promote

352 resistance to CAR-T therapy in human $B$ cell malignancies, we examined RNAseq data sets generated using pre-treatment bone marrow biopsy samples from B-ALL patients who received

354 CAR-T therapy. ${ }^{22}$ Here, we generated a sensitizer signature from the overlap of our bone marrow depleting hits and reasoned that decreased expression of this signature should

356 correlate with better outcomes in patients since these genes represent novel resistance mediators to CAR-T therapy. In line with our prediction, patients who experience complete 358 responses (CRs) show significantly less expression of this sensitization signature compared to non-responders (NRs) (Figure 6a). To further examine these patient data, we also generated a

360 JAK/STAT/MHC-I resistance signature by overlapping the top depleters in our screen with the top overexpressed genes in tumor cell cluster 2 from our transcriptional analysis. Strikingly, this

362 resistance signature is correlated with poor outcomes in CAR-T treated patients, with NRs demonstrating significantly increased expression of our JAK/STAT/MHC-I gene set compared to

364 CRs (Figure 6b). Interestingly, these data are consistent with recent reports in large B cell lymphoma ( $L B C L)$ where high intratumoral interferon signaling is correlated with poor outcomes

366 after CAR-T treatment in patients. ${ }^{44}$ Here, tumor cell expression of an interferon stimulated gene resistance signature (ISG.RS) associated with ICB resistance, is a strong predictor for CAR-T

368 treatment failure in LBCL. ${ }^{45,46}$ Further examination of this signature in our model revealed that relapsed B-ALL cells with high IFNy signaling after CAR-T failure were also enriched for

370 expression of the ISG.RS gene set (Figure 6c). Taken together, these data suggest that intratumoral JAK/STAT/IFNy signaling, along with downstream antigen processing and

372 presentation pathways, may be key determinants of CAR-T response in human B-ALL (Figure $6 d)$. 


\section{Discussion}

Despite the immense therapeutic potential of CAR-T therapy, emerging long-term follow up data has highlighted that relapse is likely to be a significant problem for most patients treated with CAR-T cells. ${ }^{5,8-13}$ Using our screening platform, we identified and validated JAK/STAT/IFNY signaling and components of the antigen processing and presentation machinery as critical in vivo mediators of CAR-T resistance in B-ALL. Notably, loss of these pathways in vitro results in either the opposite phenotype, as with JAK/STAT/IFNy signaling, or no phenotype at all, as with the $\mathrm{MHC}-\mathrm{I}$ molecule, $\mathrm{Qa}-1^{\mathrm{b}}$, highlighting the importance of assaying CAR-T function in immunocompetent models of disease. We also show that tumor cells that have lost the ability to sense IFNy, either via loss of Ifngr1 or loss of critical downstream signaling components of this pathway are also functionally null for Qa- $1^{\mathrm{b}}$. Hence, the mechanism by which JAK/STAT/IFNy signaling promotes CAR-T resistance in B-ALL is, at least in part, dependent on IFNy-induced MHC-I expression. This result is particularly surprising, given that the functionality of CAR-T cells was designed to be completely independent of antigen presentation pathways. Our data directly argue against this notion, highlighting instead a critical role for MHC-I signaling in determining CAR-T response, potentially through the engagement of inhibitory receptors on CAR-T cells themselves or on other cellular components of the immune system, most notably, 392 NK cells, in the case of Qa-1 ${ }^{\text {b }}$.

Our results also suggest that the resistance mechanisms to CAR-T therapy are quite 394 distinct from those seen in response to anti-PD1/CTLA4 immune checkpoint inhibitor therapy. Indeed, loss of JAK/STAT/IFNY signaling is an established resistance mechanism to checkpoint

396 therapy, as these agents require target cell MHC expression to promote T-cell mediated killing. For example, LOF mutations in the IFNY receptor signaling molecules JAK1/2 or the HLA class I

398 molecule $\beta$-2-microglobulin (B2M) have been shown to render tumors refractory to immune checkpoint blockade (ICB). ${ }^{47-50}$ The tumor cell strategy for CAR-T cell evasion evinced by our

400 data represents an unexpected form of therapeutic resistance, in which tumor cells are modified by the therapeutic agent, itself, to achieve a resistant state. Specifically, B-ALL sensing of IFNy

402 in the proinflammatory microenvironment following CAR-T treatment leads to JAK/STAT mediated induction of factors that promote CAR-T resistance. This form of adaptive resistance

404 suggests that tumor-specific targeting of JAK/STAT signaling or key downstream pathways may render B-ALL tumors sensitive to CAR-T cells that persist in an activated state after relapse.

406 Furthermore, these data suggest that adaptation to CAR-T therapy may confer a collateral 


\section{Ramos, Koch, and Liu et al: Confidential}

sensitivity to immunotherapies that target tumor cells induced to express high levels of surface $408 \mathrm{MHC}$.

Lastly, our results also have implications for patient stratification in CAR-T therapy. We

410 show that elevated expression of a JAK/STAT/MHC-I signature in pre-treatment patient samples is associated with poor outcomes in CAR-T therapy, raising the possibility that this

412 transcriptional program may delineate a group of patients that harbor B-ALL tumors with some amount of intrinsic CAR-T resistance. Given our data, it is tempting to speculate that harboring

414 leukemia cells primed for effective IFNY sensing and downstream signaling sets the stage for therapeutic failure after B-ALL cells are exposed to high levels of this cytokine in the

416 proinflammatory, CAR-T treated microenvironment. However, the generation and examination of larger data sets from patients receiving CAR-T therapy will be required to more fully examine

418 this hypothesis. Ultimately, our data highlight novel strategies that could be exploited for both design of improved CAR-T treatment regimens with preexisting CAR-T products, and for the

420 development of pre-treatment tumor biomarkers that better predict which patients are most likely to benefit from this promising immunotherapy.

\section{Methods}

\section{Pooled sgRNA screening}

A custom genome-wide library divided into 48 sub pools was generated in collaboration with the

426 Broad Institute's Genetic Perturbation Platform (GPP). In total, 97,336 unique guides targeting the protein coding regions of 21,958 unique murine genes with 4 sgRNAs each (plus control non-

428 targeting and intragenic cutting guides) were included. All protein coding murine genes were subdivided into 48 pools by their initial KEGG term (obtained using KEGG REST API in BioPython,

430 biopython.org), in a non-redundant manner. All four guides targeting the protein coding region of any given gene were kept together in the same pool. Using this approach, only $36 \%$ of protein-

432 coding genes could be classified into a KEGG pathway. Thus, the first 14 sub pools and part of sub pool 15 were filled by KEGG genes. All other genes were randomly distributed among the

434 remaining sub pools. Mouse essential genes (defined as orthologous mouse genes for the human essential gene set from Hart et al., 2015, 1530 genes; obtained from Ensembl Biomart) were

436 divided evenly across all pools. Guides against human EGFRvIII, human CD22, human CD19, and murine Cd19 were included in the first pool. ${ }^{51}$ Guides against olfactory genes $(1,133$ total)

438 were also distributed evenly amongst all sub pools. All 48 sub pools were cloned into a lentiviral pRDA-Crimson_170 vector (Figure 10). To preserve library complexity, a minimum of 1000-fold 440 coverage of the sgRNA library was maintained at each in vitro step before the screen, and at a 
minimum of 150 -fold coverage (range: 153 to 203-fold coverage in vivo, all in vitro screens were 442 performed above 500x) was maintained in all screens completed. Pool A consisted of the first 8 sub pools and had a total of 15,308 sgRNAs targeting the protein coding regions of 3,648 unique

444 mouse genes. Pool B consisted of sub pools 9-16 and had a total of 15,147 sgRNAs targeting the protein coding regions of 3,648 unique mouse genes. Pool C consisted of sub pools 17-24 and 446 had a total of $15,258 \mathrm{sgRNAs}$ targeting the protein coding regions of 3,648 unique mouse genes. Pool D consisted of sub pools 25-32 and had a total of 17,335 sgRNAs targeting the protein 448 coding regions of 3,648 unique mouse genes. Pool E consisted of sub pools 33-40 and had a total of 14,713 sgRNAs targeting the protein coding regions of 3,648 unique mouse genes. Pool

$450 \mathrm{~F}$ consisted of sub pools $41-48$ and had a total of 19,575 sgRNAs targeting the protein coding regions of 3,718 unique mouse genes. Cloned and sequenced plasmid pools, and viral 452 supernatant were generated by the Broad Institute's GPP.

454 For screens, Cas $9^{+}$cells were thawed, recovered, and expanded for 5 days to ensure robust growth, and then tested for cutting efficiency to ensure high rates of editing efficiency. ${ }^{27,52}$ After

456 cutting assays were completed, cells were expanded over three additional days and infected with sub pools. For each of the 48 sub pools, $6 \times 10^{7}$ cells were spin-infected with predetermined

458 amounts of viral supernatant (determined using titration experiments, data not shown), such that $15-30 \%$ of all cells were infected (expressed E2-Crimson, and survived puromycin selection;

$460 \mathrm{MOI}<<1)$. The resulting cells were resuspended at a concentration of $10^{6} \mathrm{cells} / \mathrm{mL}$ in viruscontaining medium supplemented with $10 \mu \mathrm{g} / \mathrm{mL}$ polybrene (Sigma), divided into 6-well plates,

462 and centrifuged at $1000 \mathrm{xg}$ and $37^{\circ} \mathrm{C}$ for $1.5 \mathrm{hrs}$. Cells were then pooled into flasks and cultured overnight. Thirty-six hours later, cell density was adjusted to $10^{6}$ cells $/ \mathrm{mL}$ (and was never allowed

464 to go over $3 \times 10^{6}$ cells $\left./ \mathrm{mL}\right)$ and puromycin selection (2 $\mu \mathrm{g} / \mathrm{mL}$, Gibco, A1113803) was started. Cells were selected over two days and then spun out of puromycin-containing medium. Cells were

466 then allowed to recover for one day, after which the appropriate number of infected, selected, and recovered cells were flow cytometry sorted and combined into their respective pools. The next

468 day, cells from large, combined pools were prepared for tail vein injection into mice or kept in culture for the in vitro screens. Two days later, CAR-T cells were adoptively transplanted into mice

470 via tail vein injection at the indicated doses. For all in vitro screens, $1.4 \times 10^{7}$ library cells were seeded and treated two days later (on the same schedule as mice) with control CAR-T cells (anti-

472 hEGFRvIII), anti-mCD19 CAR-T cells, or with no CAR-T cells, at E:T ratios indicated in the text. In vitro CAR-T screens were set up in triplicate while the no CAR-T condition was kept in a single 474 plate. Input samples were collected just after puromycin selection had completed (Input PS) and 


\section{Ramos, Koch, and Liu et al: Confidential}

on the day cells were injected into mice/set up for in vitro screens (Input or Input DOI). Upon 476 becoming moribund, mice were sacrificed and E2-Crimson ${ }^{+}$cells were sorted from the bone marrow and spleen (average number sorted cells per compartment $=1.38 \times 10^{7}$ ). For in vitro

478 screens where the B-ALL cells viability was above $95 \%$ and represented more than $99 \%$ of all cells present in the sample, cells were counted and $2 \times 10^{7}$ cells were collected for gDNA isolation.

480 The only condition that did not meet this cutoff was the anti-mCD19 at E:T of 1:2 for all 6 pools. Here, CAR-T cells were completely gone from culture, but B-ALL viability of cells remained low at

$48230-60 \%$ across replicates, necessitating sorting to isolate cells $\left(1.4-2.0 \times 10^{7}\right.$ sorted per replicate, per condition).

Finally, gDNA from all cells were isolated using the Machery Nagel L Midi NucleoSpin Blood Kit 486 (Clontech, 740954.20). Modifications to the manufacturer's instructions were added as follows: in step 1, cells were lysed in the kit's proteinase $\mathrm{K}$ containing lysis buffer for longer (overnight at $48870^{\circ} \mathrm{C}$ ). The next morning, lysates were allowed to cool to room temperature, $4.1 \mu \mathrm{L}$ of RNase $\mathrm{A}$ (20 mg/mL; Clontech, 740505) was added, and cells were incubated for 5 minutes at room 490 temperature. The procedure then continued as indicated by the manufacturer. PCR inhibitors were removed from the resulting gDNA (Zymo Research, D6030) and the concentration of the 492 resulting gDNA was measured using the Qubit dsDNA HS assay kit (ThermoFisher, Q32854), and if necessary, diluted to $200 \mathrm{ng} / \mu \mathrm{L}$ with elution buffer. gDNA was then submitted for Illumina 494 sequencing.

\section{Screen hit discovery}

Sample quality control was performed by counting the number of gRNA sequences that show at least 30 reads in each sample. Samples with more than $30 \%$ of gRNA sequences that were filtered out after the above procedure were excluded from further analyses. Guide RNA-level read counts were scaled by total read count for each sample and logarithm-transformed. Gene-level enrichment and depletion scores were computed by averaging log-normalized read counts across

502 all gRNA sequences against each gene. Six different pooled libraries were aggregated targeting a total of 21,958 unique murine genes with a total of 88,793 sgRNAs (4 per gene plus non-

504 targeting and intergenic-cutting controls). Input samples were used as a baseline for computing scores. Specifically, every gene was first assigned an 'essentiality score' computed as the 506 difference (i.e. log-fold change) between the average gene-level counts for the gene across replicates and that in the input sample. The CAR-T therapy enrichment/depletion score for a given

508 gene is computed as the difference between the essentiality scores of that gene in anti-mCD19 
CAR-T cell treated samples and in anti-hEGFRvIII CAR-T cell treated samples. For sample

510 groups that have at least 2 samples retained after quality control, Student's t-tests were performed to obtain p-values for assessing significance of difference between the scores in the treatment

512 group and the control group.

\section{Bulk transcriptome profiling and analysis}

Total RNA was extracted from $1 \times 10^{6}$ cells per sample using the Macherey-Nagel Nucleospin

516 RNA Plus kit, and RNA sample quantity and quality was confirmed using an Agilent Fragment Analyzer. RNAseq libraries were created from 250ng of total RNA using the NEBNext Ultrall

518 Directional RNA Library Prep kit (New England Biolabs) using half volume reagents and 14 cycles of PCR. Illumina library quality was confirmed using the Fragment Analyzer and qPCR and

520 sequenced on an Illumina NextSeq500 using v2.1 chemistry with 40nt paired end reads (RTA version 2.11.3). Paired-end RNA-seq data was used to quantify transcripts from the mm10 mouse 522 assembly with the Ensembl version 101 annotation using Salmon version 1.3.0. ${ }^{53}$ Gene level summaries were prepared using tximport version $1.18 .0^{54}$ running under $\mathrm{R}$ version $4.0 .3 .{ }^{45}$

\section{Single cell transcriptome profiling and data processing}

526 Mice were transplanted with $3 \times 10^{6} \mathrm{~B}-\mathrm{ALL}$ cells and challenged with $10^{7} \mathrm{CAR}-\mathrm{T}$ cells targeting either CD19 or a control epitope (human EGFRvIII). Upon becoming moribund, mice were

528 sacrificed and B-ALL cells were sorted from the bone marrow and spleen. For each sample, approximately 10,000 live B-ALL cells were sorted for transcriptional profiling. Single-cell

530 expression libraries were prepared using the 10x Genomics Chromium v3 reagents. Sequencing data was aligned to the $\mathrm{mm} 10$ reference genome and converted to fastq files using bcl2fastq

532 (v2.20.0.422). Cell count matrices were generated using cellranger (v.5.0). Matrices were analyzed by Seurat (v4.0.4) for R (v4.0.2). Digital gene expression matrices were filtered to 534 exclude low quality cells (< $1000 \mathrm{UMI}$, < 400 genes or $>8000$ genes, $>50 \%$ mitochondrial reads). Low-quality cells were further filtered from the dataset using the variance sink method as 536 previously described. ${ }^{55}$ Briefly, data was normalized and scaled, known cell cycle genes were regressed out. ${ }^{56}$ Principal component analysis was performed on regressed and scaled data.

538 Standard deviation of principal components was quantified using an elbow plot, input dimensions for SNN clustering (EGFRv3 bone marrow $=35$, EGFRv3 spleen $=42$, CD19 bone marrow $=30$,

540 CD19 spleen $=30$ ) at which standard deviation $=2$. SNN clustering was performed to generate UMAP plots (k.param $=40$, res $=0.5)$. Clusters containing low quality cells $(50 \%$ of cells with $>$ $54210 \%$ mitochondrial reads) were removed from the dataset. After filtering, samples from bone 
marrow and spleen treated with EGFRv3-CAR-T or CD19-CAR-T were merged into a single

544 dataset. Cell cycle phase was assigned using the cell cycle scoring function based on expression of known cell cycle genes. ${ }^{56}$ Merged data set was normalized and scaled, cell cycle genes were

546 regressed out. Principal component analysis was performed, and standard deviation of principal components was quantified by elbow plot. Nearest neighbors were found $(\mathrm{dim}=50$, k.param $=$

548 40) then clustered using SNN clustering (res = 0.5). Enriched genes for each cluster were identified with the cluster marker function. Cluster occupancy was quantified for each treatment

550 condition and phase of the cell cycle to further define therapeutic response.

\section{Mouse maintenance and studies}

All mouse experiments were conducted under IUCAC-approved animal protocols at the

554 Massachusetts Institute of Technology. The mouse strains used in this study included C57BL/6 (Jackson) and NOD/SCID/IL2Rg ${ }^{-/-}$(NSG; Jackson Laboratory). Immunocompetent recipient mice

556 were sub-lethally irradiated $(1 \times 5 \mathrm{~Gy})$ immediately prior to transplantation with B-ALL cells, receiving ACT of CAR-T cells 2 days later, as noted in the text. For in vivo screens, mice were

558 injected with $3 \times 10^{6}$ Cas9 $9^{+}$library B-ALL cells and the indicated number of CAR-T cells. Both BALL and CAR-T cells were prepared for transplantation by resuspending in $200 \mu \mathrm{L}$ Hank's 560 balanced salt solution (Lonza) and loaded in 27.5-gauge syringes (Becton Dickinson). All cell solutions were administered via tail vein injections. For in vivo blocking of IFNY, mice were injected

562 i.p. every third day with $200 \mu$ g of InVivoMAb anti-mouse IFNy antibody (clone XMG1.2, Bio X Cell) or $200 \mu \mathrm{g}$ of InVivoMAb anti-horseradish peroxidase control antibody (clone HRPN, Bio X

564 Cell), starting the day after ACT. For in vivo inhibition of JAK1/2, Ruxolitinib (Selleckchem, INCB018424) was resuspended according to manufacturer guidelines and mice were dosed 566 every twelve hours via oral gavage with $90 \mathrm{mg} / \mathrm{kg}$ Ruxolitinib or vehicle control, starting the day after disease transplantation.

\section{Bioluminescence studies}

570 XenoLight D-Luciferin Potassium Salt D (PerkinElmer, 122799) was used for standard bioluminescent imaging (resuspended at $30 \mathrm{mg} / \mathrm{mL}$ in saline, sterile filtered, and stored at $-80^{\circ} \mathrm{C}$ ).

572 Mice were weighed and luciferin was loaded in 27.5-gauge syringes (Becton Dickinson) and administered via intraperitoneal injection at a dose of $165 \mathrm{mg} / \mathrm{kg}$. Mice were then anesthetized

574 with 2.5\% isoflurane (Piramal Critical Care, NDC\# 66794-013-25), delivered at $1 \mathrm{~L}$ per minute in $\mathrm{O}_{2}$. Ten minutes from the time of luciferin injection, animals were imaged on a Xenogen IVIS

576 system at consistent exposures between groups with small binning. Data was analyzed using 
Living Image version 4.4 software (Caliper Life Sciences). Images were normalized to the same 578 color scale for figure generation.

\section{Cell culture}

All cell lines were mycoplasma negative.

582 Murine B-ALL cells: Cells were cultured in RPMI with L-glutamine (Corning, 10-040-CM), supplemented with $10 \%$ fetal bovine serum and 2-mercaptoethanol to a final concentration of

$5840.05 \mathrm{mM}$ (Gibco, 21985023).

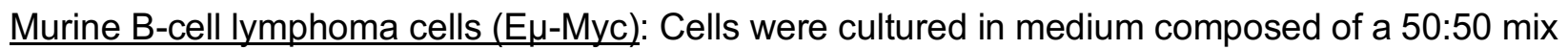

586 of IMDM with L-glutamine and 25mM HEPES (Corning, 10-016-CM) and DMEM with L-glutamine and sodium pyruvate (Corning, 10-013-CM), supplemented with 10\% FBS and 2-

588 mercaptoethanol to a final concentration of $0.05 \mathrm{mM}$ (Gibco, 21985023).

Murine T cells: $T$ cells harvested from the spleens of mice were cultured in plates coated with

590 activating antibodies (as described in CAR-T cell production methods) in T cell medium (TCM): RPMI with L-glutamine (Corning, 10-040-CM), supplemented with 10\% FBS, recombinant human

592 IL-2 (rhIL-2, final concentration of $20 \mathrm{ng} / \mathrm{mL}$; Peprotech, Cat\# 200-02-1mg), and 2mercaptoethanol to a final concentration of $0.05 \mathrm{mM}$ (Gibco, 21985023).

594 Human cell lines: 293T and Raji cells were all mycoplasma negative. 293T cells are cultured in DMEM with L-glutamine and sodium pyruvate (Corning, 10-013-CM) supplemented with 10\% 596 FBS. Raji cells were cultured in RPMI with L-glutamine (Corning, 10-040-CM) supplemented with $10 \%$ FBS.

\section{Viral supernatant production}

600 Viral supernatant was produced using standard methods. Briefly, 293T cells were transfected with retroviral or lentiviral transfer plasmid and packaging vector (retrovirus: pCL-Eco, Addgene, 602 12371; lentivirus: psPAX2, Addgene, 12260 with VSVg envelop plasmid pMD2.G, Addgene, 12259) using Mirus TransIT-LT1 (Mirus, MIR2305) as indicated by the manufacturer. The next

604 day, 293T cells were switched into medium composed of 60\% RPMI complete and 40\% DMEM complete. Viral supernatant was collected 24 and 48 hours after transfection, passed through a

6060.45 um filter to remove residual $293 \mathrm{~T}$ cells, and kept at $4^{\circ} \mathrm{C}$ for a maximum of 4 days.

\section{In vitro killing assay}

In vitro CAR-T killing assays were performed using standard methods. ${ }^{57,58}$ Briefly, target cells are 610 counted and co-cultured with or without CAR-T cells at indicated E:T ratios (accounting for CAR- 
T infection rate) in RPMI complete supplemented with 2-mercaptoethanol and 10\% FBS but no

612 rhlL-2. Sixteen to twenty-four hours later, the total cell number per well was counted and the cell suspension was analyzed by flow cytometry to assess for live/dead, \%mCD $19^{+}$cells, and \%CD8 ${ }^{+}$

614 cells. The densities of each cell type (CAR-T, target cell, non-transduced T cell) were also determined via flow cytometry. The resulting target cell densities in CAR-T containing wells are

616 then normalized to the resulting target cell density in control wells seeded with the same number of target cells but without CAR-T cells.

\section{Interferon gamma ELISA release assay}

620 Standard methods were used for enzyme-linked immunosorbent assay (ELISA). Briefly, supernatant from in vitro CAR-T killing assays was collected and spun down to remove any 622 contaminating cells. IFNY released into the supernatant by CAR-T cells is then measured using the DuoSet ELISA kit for mouse IFNy (R\&D systems, DY485) and Nunc MaxiSorp flat bottom

624 plates (Thermo Fisher Scientific, 44-2404-21) on a Tecan infinite 200 Pro machine, as indicated by the manufacturer. To ensure that the assay is completed within the linear range of the kit,

626 supernatant is initially diluted 1:10 in reagent diluent. At least six serial 4-fold dilutions are then performed. At least one standard curve for this assay is generated per plate and at least two

628 standard curves for the entire experiment are constructed using standard solutions supplied by the manufacturer. Substrate solution used is 1-Step ${ }^{T M}$ Ultra TMB-ELISA (Thermo Fisher

630 Scientific, 34028) and stop solution used is sulfuric acid 2N stop solution (VWR, BDH7500-1). Bovine serum albumin (BSA; Sigma, A8022-500G) is prepared as a sterile filtered $5 \%$ stock in

632 PBS (Corning, 21-031-CV).

634 CAR-T cell production

Before collecting T cells, 6-well plates were coated overnight with activating antibodies against 636 mCD3e (Bio X-Cell, BE0001-1) and mCD28 (Bio X-Cell, BE0015-1) at $5 \mu \mathrm{g} / \mathrm{mL}$ each in PBS (Corning, 21-031-CV) at $4^{\circ} \mathrm{C}$. The next day, 8-12-week-old male C57BL/6 mice (Jackson) were 638 sacrificed, and their spleens were collected. $\mathrm{CD}^{+} \mathrm{T}$ cells were isolated using Miltenyi Biotec CD8a (Ly-2) MicroBeads for mouse (positive selection kit; Miltenyi, 130-117-044) and LS columns

640 (Miltenyi, Cat\# 130-042-401) as indicated by the manufacturer. Coated plates were rinsed once with PBS and T cells were resuspended at $0.5 \times 10^{6}$ to $10^{6}$ cells $/ \mathrm{mL}$ in T cell medium (TCM, recipe

642 in cell culture methods). After 24 hours, activated T cells were collected and placed into fresh TCM after counting. Cell concentrations were then adjusted to $10^{6}$ cells $/ \mathrm{mL}$ in a 50:50 mix of 644 TCM:retroviral (RV) supernatant supplemented with protamine sulfate to a final concentration of 
$10 \mu \mathrm{g} / \mathrm{mL}$ (MS Biomedicals, ICN19472910), 2-mercaptoethanol to a final concentration of 0.05 $646 \mathrm{mM}$, and rhIL-2 to a final concentration of $20 \mathrm{ng} / \mathrm{mL}$. Once resupended, cells were spin-infected at $1000 \mathrm{xg}$ for $1.5 \mathrm{hrs}$ at $37^{\circ} \mathrm{C}$ on new antibody coated plates. The next day, T cells were collected

648 from plates, resuspended in fresh TCM at a cell density of $0.5 \times 10^{6}$ to $10^{6}$ cells $/ \mathrm{mL}$, and re-plated on new antibody-coated plates. Twenty-four hours later, T cells were collected from antibody

650 coated plates and prepared for tail vein injection into animals or for in vitro kill assays/screens, as described above. T cells were always cultured and infected on PBS rinsed, antibody-coated 6-

652 well plates, as described above, except during in vitro killing assays where no activating antibodies were ever used.

\section{Western Blotting}

656 Cells were lysed with RIPA buffer (Boston BioProducts, BP-115) supplemented with 1X protease inhibitor mix (cOmplete EDTA-free, 11873580001, Roche). Protein concentration of cell lysates

658 was determined using Pierce BCA Protein Assay (ThermoFisher Scientific, 23225). Total protein $(40-60 \mu \mathrm{g})$ was separated on 4-12\% Bis-Tris gradient SDS-PAGE gels (Life Technologies) and

660 then transferred to PVDF membranes (IPVH00010, EMD Millipore) for blotting.

\section{Plasmids, cloning, and sgRNAs}

Packaging and envelope plasmids used for viral production

664 Retrovirus: pCL-Eco (Addgene, 12371)

Lentivirus: psPAX2 (Addgene, 12260) with VSVg envelop plasmid pMD2.G (Addgene, 12259) or

666 pCMV-EcoEnv (Addgene, 15802)

Chimeric Antigen Receptor (CAR) plasmids

668 The murine CD19 targeting second generation CAR 1D3-28Z.1-3 containing inactivating mutations in the $1^{\text {st }}$ and $3^{\text {rd }}$ ITAM regions of the CD3- $\zeta$ chain was synthesized by Twist Bioscience

670 and cloned into the GFP ${ }^{+}$MP71 retroviral vector. ${ }^{28,59}$ The clinically used scFv sequence (heavy chain linked to light chain variable regions) against human CD19, FMC63, was provided by the

672 Maus lab. A CD28-containing $2^{\text {nd }}$ generation murine CAR targeting hCD19 protein was then constructed by switching out the scFv for 1D3-28Z.1-3 in the anti-mCD19 CAR and replacing it

674 with the FMC63 scFv (Twist Bioscience). The same technique was used for the 3C10 scFv targeting human EGFRvIII, which was reported by the Rosenberg lab. ${ }^{60}$ All CAR constructs are

676 identical, containing a CD8a leader sequence, followed by the respective scFv, followed by an IgG4 hinge sequence, a portion of the murine CD28 molecule from amino acids IEFMY to the 3'

678 terminus, and finally, the cytoplasmic region of the murine CD3- $\zeta$ chain from amino acids RAKFS 
to the $3^{\prime}$ terminus with both tyrosines in ITAMs 1 and 3 mutated to phenylalanines as described

680 (all in frame). ${ }^{28}$ All CAR constructs were extensively tested to ensure that they only targeted their peptide of interest. Human EGFRvIII expression was induced using pMSCV-XZ066-EGFRvIII

682 (Addgene, plasmid 20737) and murine hEGFRvIII ${ }^{+}$B-ALL cells were generated. Retroviral supernatant to induce hCD19 expression was provided by the Maus lab. All CARs were

684 extensively tested in vitro (killing assays methods) and in vivo (as described in text) to ensure no off-target effects were present.

686 CRISPR plasmids

To generate Cas9 ${ }^{+}$murine B-ALL cell lines, lentiCas9-Blast (Addgene, 52962) was used, and 688 cells were selected with Blasticidin (Gibco, A1113903) at $20 \mu \mathrm{g} / \mathrm{mL}$ for 7 days and then single cell cloned and assayed for Cas9 expression via WB. Guide RNAs for murine Cd19 were designed using the Broad Institute's sgRNA Designer tool (https://portals.broadinstitute.org/gppx/crispick/public) and cloned into lentiGuide-Puro (Addgene,

692 52963) for the functional cut assay (tracking loss of mCD19 on the cell surface) or pRDACrimson_170 to generate KOs of various test genes (vector testing, data not shown). ${ }^{27}$ Guide

694 RNAs used are below (Forward/Reverse):

Murine Cd19 sgRNAs:

696

698 sgRNA\#42, targets exon 6 (5'-CACCGAATGACTGACCCCGCCAGG)/(5'AAACCCTGGCGGGGTCAGTCATTC) sgRNA\#43, targets exon 2 (5'-CACCGCAATGTCTCAGACCATATGG)/(5'AAACCCATATGGTCTGAGACATTGC)

700 Other plasmids

MSCV-mCherry (Addgene, 52114) was used to generate 20.12DP cells from mCherry GFP $^{+}$ $702 \quad 20.12$ cells.

704 Generating Qa-1 ${ }^{\text {b }}$ knockout B-ALL cells

B-ALL cell lines deficient for $\mathrm{Qa}-1^{\mathrm{b}}$ were generated using CRISPR-Cas9 technology. Single guide

706 RNAs directed against the H2-T23 locus were designed using the Broad Institute's sgRNA Designer tool and the sgRNA sequence (5'-GTACTACAATCAGAGTAACGA-3') was cloned into 708 pRDA-Crimson_170, as described above. Cas9 ${ }^{+}$B-ALL cells (clone RH62) were transduced with sgRNAs and selected with puromycin over 48 hours. Loss of Qa- $1^{\mathrm{b}}$ was confirmed by incubating

710 transduced cells with $30 \mathrm{IU} / \mathrm{mL}$ IFNy for 24 hours and subsequently analyzing Qa- $1^{\mathrm{b}}$ surface expression by flow cytometry, as previously described. $.^{42} \mathrm{Qa}-1 \mathrm{~b}^{-}$cells were then FACS sorted

712 twice until a pure population of $\mathrm{Qa}-1^{\mathrm{b}}$ knockout cells was established. 


\section{$714 \quad$ Antibodies}

Western blotting: anti- $\beta-A C T I N$ (Cell signaling, 4967S), anti-CD19 (Abcam, ab25232), anti-Cas9

716 (ActiveMotif, 61577), anti-JAK2 (Cell Signaling Technology, 3230), anti-IFNyR1/CD119 (R\&D Systems, MAB10261), anti-STAT1 (Cell Signaling Technology, 9172), anti-rabbit IgG HRP-linked

718 antibody (Cell Signaling Technology, 7074), anti-mouse lgG HRP-linked antibody (Cell Signaling Technology, 7076), anti-rat IgG HRP-linked antibody (Cell Signaling Technology, 7077), Rabbit

720 anti-Armenian Hamster lgG H\&L (HRP) (Abcam, ab5745).

722 Flow cytometry: anti-mouse CD19-BV785 (BioLegend, 115543), anti-mouse CD8-PE/Cy7 (BioLegend, 100722), anti-human CD19-APC/Cy7 (BioLegend, Cat\# 302218), anti-mouse Qa-1 ${ }^{\text {b_ }}$

724 BV786 (BD Biosciences, 744390).

\section{Statistical analysis}

All Statistical analyses were performed using GraphPad Prism 9 (GraphPad Software Inc). The

728 specific statistical tests performed are specified in figure legends. Differences are considered significant for P-values $\leq \mathbf{0 . 0 5}$, or as indicated when adjustments for multiple hypothesis testing 730 was required.

732 Data Availability

RNA sequencing data generated in this manuscript is in the process of being uploaded to

734 publicly accessible online repositories. Sequencing data from all screens are available from the corresponding author on reasonable request. All other data are included within the manuscript

736 figures or as supplementary information.

\section{Acknowledgements}

The authors thank Hojun Li, Khloe Gordon, and members of the Hemann and Vander Heiden

740 labs for valuable discussions and intellectual input, the Koch Institute's Robert A. Swanson (1969) Biotechnology Center for technical support, specifically the Flow Cytometry and

742 Preclinical Imaging and Testing facilities, and S. Levine from the MIT BioMicro Center for informative discussions about guide library and scRNA sequencing. The authors also thank

744 Beatrice Grauman-Boss for her support generating CAR virus. This work was generously supported by the MIT Center for Precision Cancer Medicine, the Ludwig Center at MIT, a

746 Margaret A. Cunningham Immune Mechanisms in Cancer Research Fellowship, a David H. 
Koch Graduate Fellowship, and the Paul and Daisy Soros Fellowship for New Americans. This

748 work was also supported in part by NCI R01-CA233477, R01-CA226898 and NIH/NIAID R21Al151827 to M.T. Hemann and the Koch Institute Support (core) Grant P30-CA14051 from

750 the $\mathrm{NCl}$.

\section{Author information}

Please note that Aviv Regev's initials are noted below as AV to differentiate them from those of

754 co-author Azucena Ramos.

Contributions

$756 \mathrm{AR}, \mathrm{YL}$, and MTH conceived the idea for the study. AR, YL, CK, and MTH designed the study. $\mathrm{AR}, \mathrm{YL}, \mathrm{CK}, \mathrm{RH}$, and JF conducted experiments. $\mathrm{RH}$ provided support with characterization of

758 clone 20.12 and clone RH62, and the entirety of the screens. RCL provided support generating clone 20.12. TK and RCL provided critical support in designing the CAR constructs. TK cloned

760 all CAR constructs. YL and DG analyzed scRNAseq data. AV, JGD, MM, and MEB gave vital advice and provided reagents. AR and $Y L$ wrote the manuscript. All authors reviewed and

762 edited the manuscript.

764 Corresponding author: Michael T. Hemann - hemann@mit.edu 
Figure 1
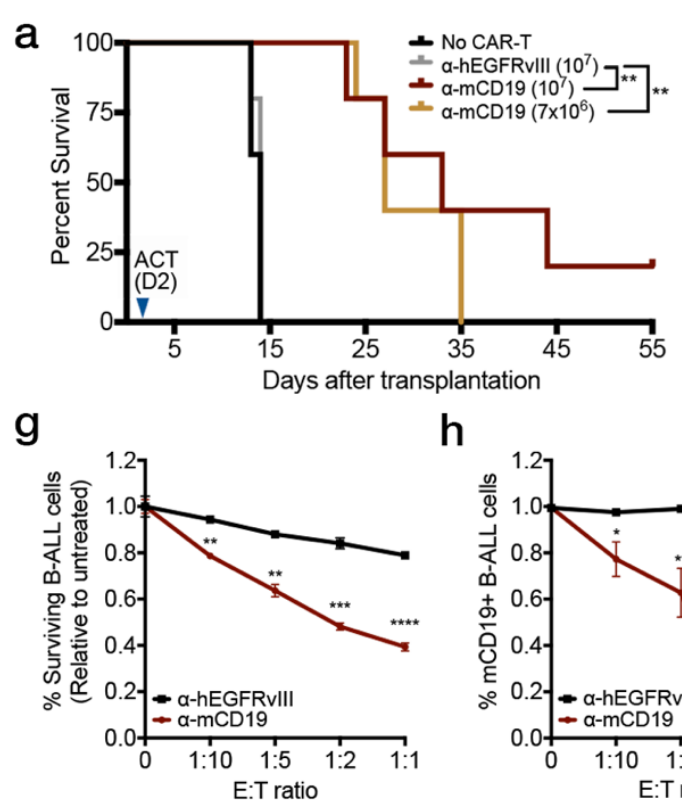

h

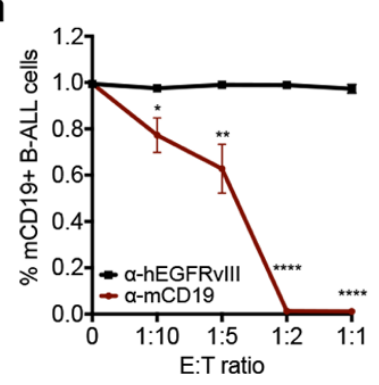

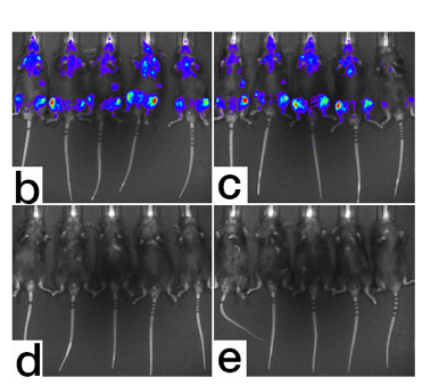

i

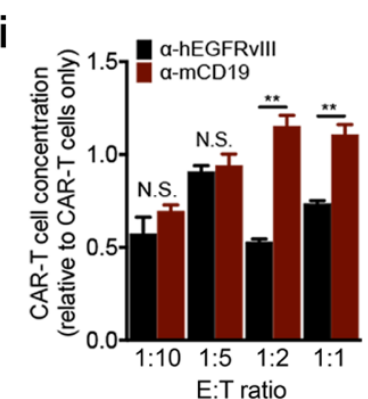

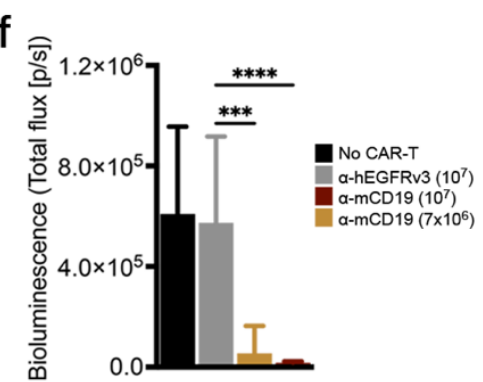

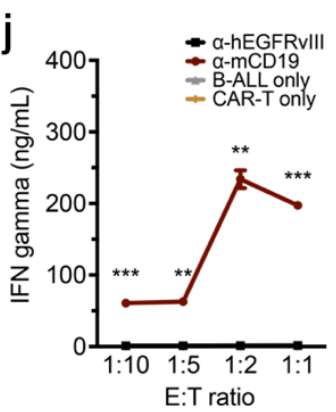

k
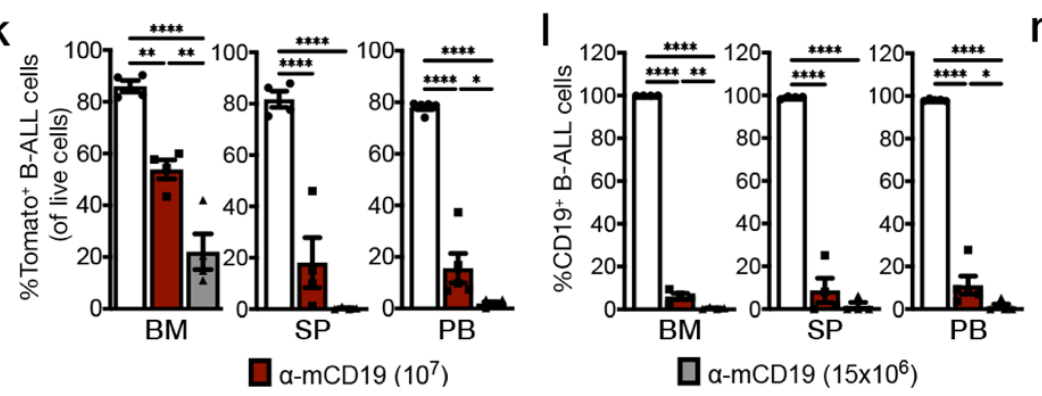

प a-mCD19 $\left(15 \times 10^{6}\right)$

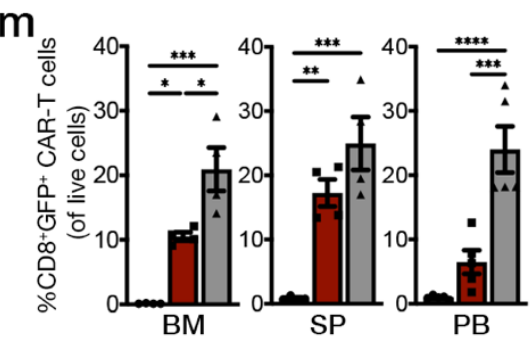

a-hEGFRvill $\left(15 \times 10^{6}\right)$

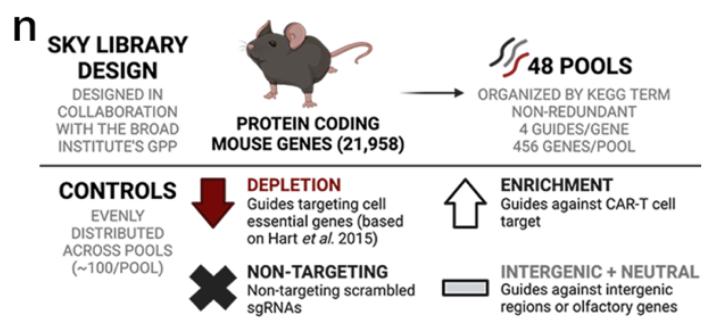

0

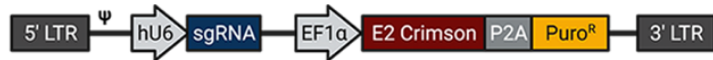

$\mathrm{p}$

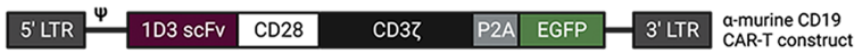

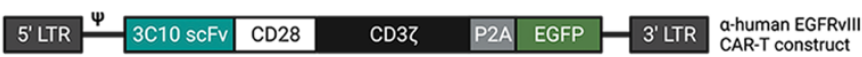

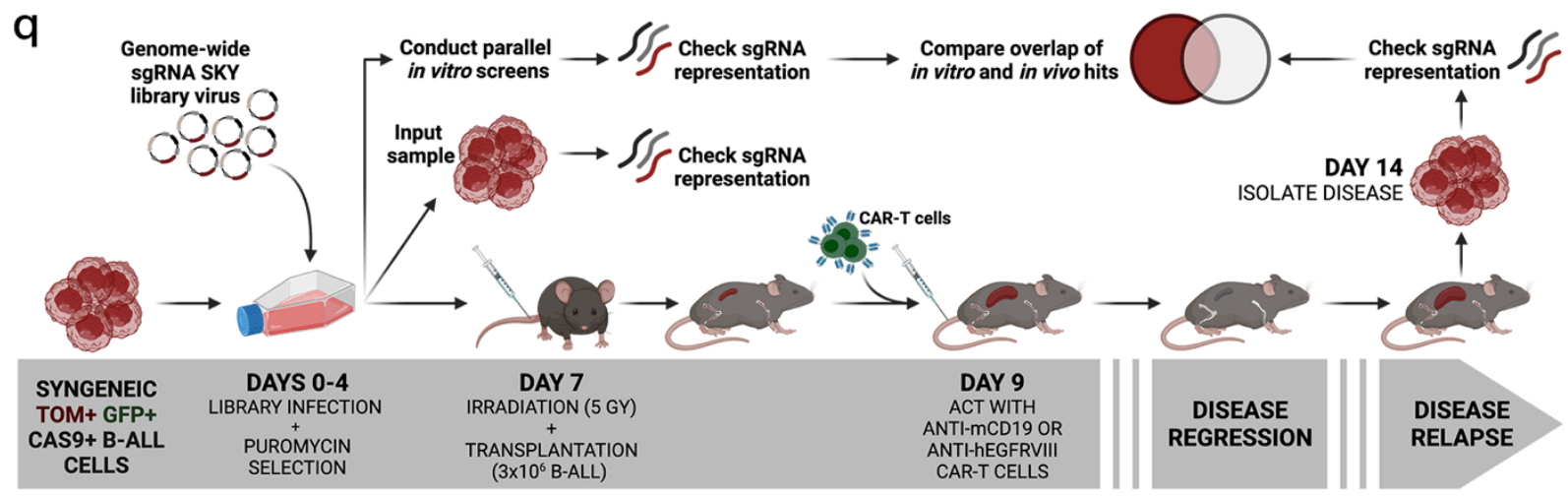


bioRxiv preprint doi: https://doi.org/10.1101/2022.02.15.480217; this version posted February 17,2022 . The copyright holder for this preprint (which was not certified by peer review) is the author/funder. All rights reserved. No reuse allowed without permission.

\section{Ramos, Koch, and Liu et al: Confidential}

Figure 2

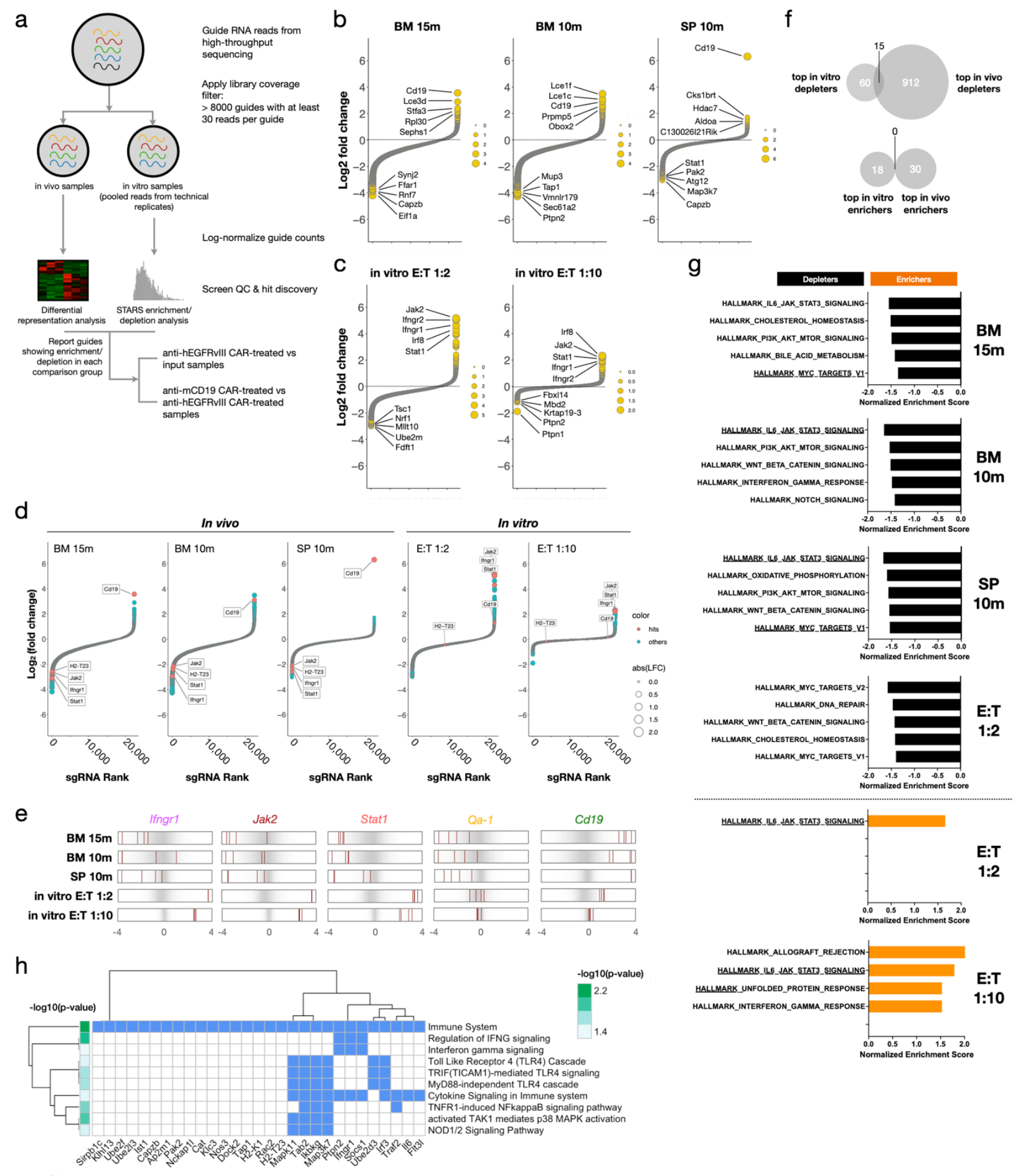


bioRxiv preprint doi: https://doi.org/10.1101/2022.02.15.480217; this version posted February 17, 2022. The copyright holder for this preprint (which was not certified by peer review) is the author/funder. All rights reserved. No reuse allowed without permission.

\section{Ramos, Koch, and Liu et al: Confidential}

Figure 3

a

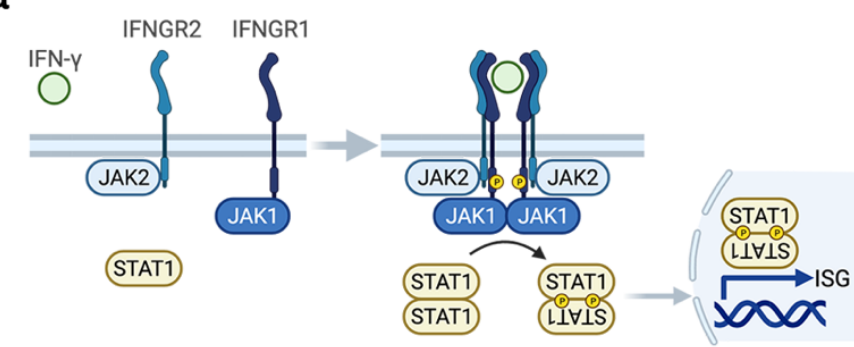

C

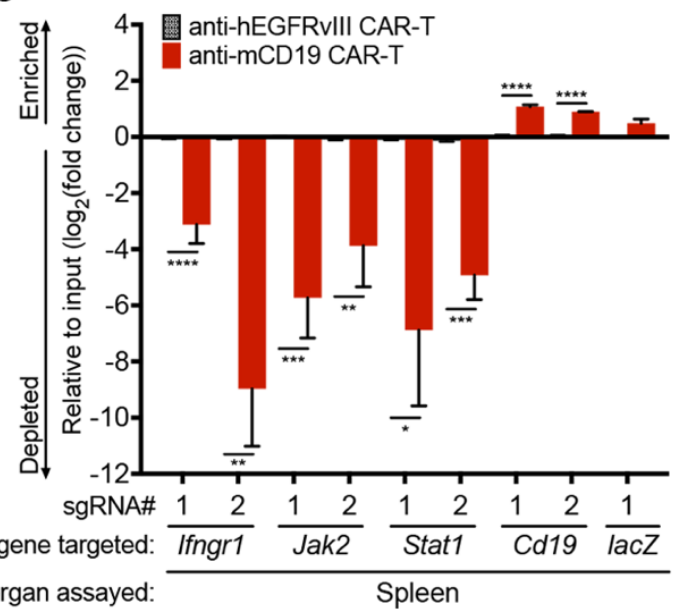

b

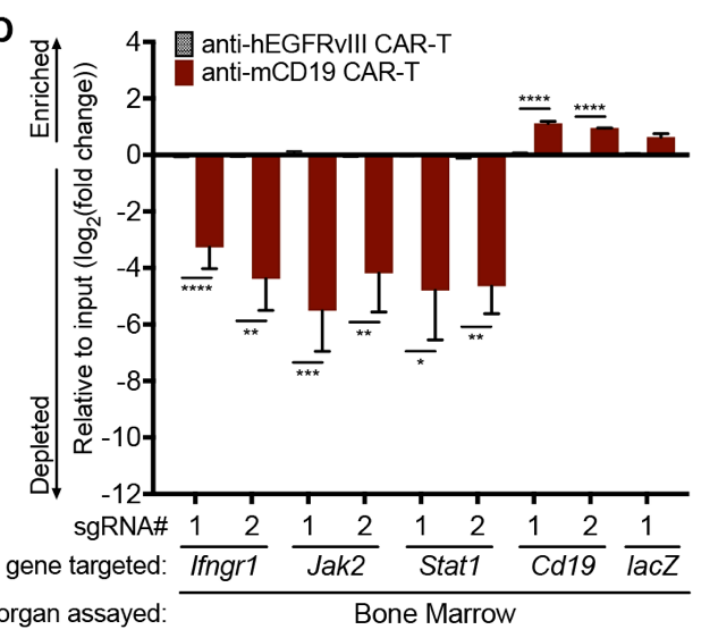

d

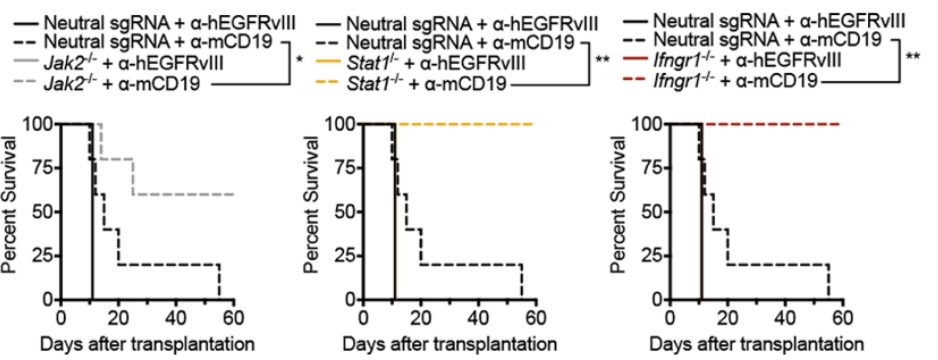

e

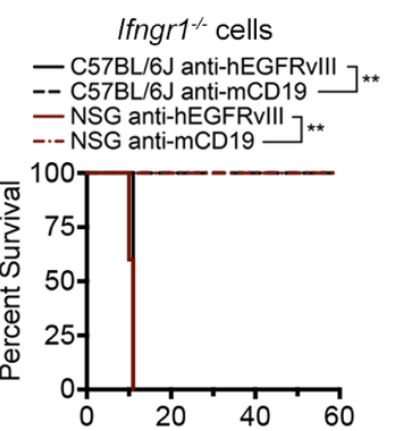

Days after transplantation

h
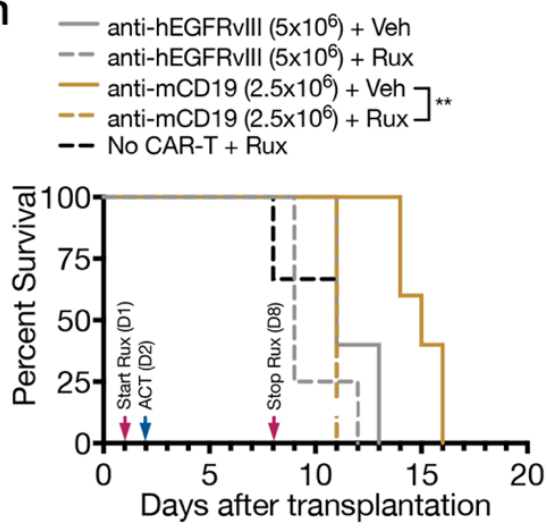

g f

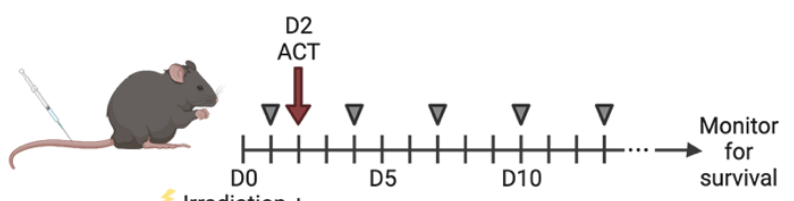

Irradiation +

Transplantation

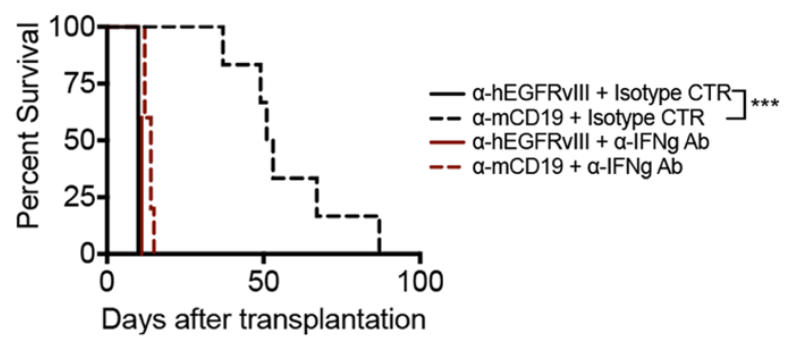

i

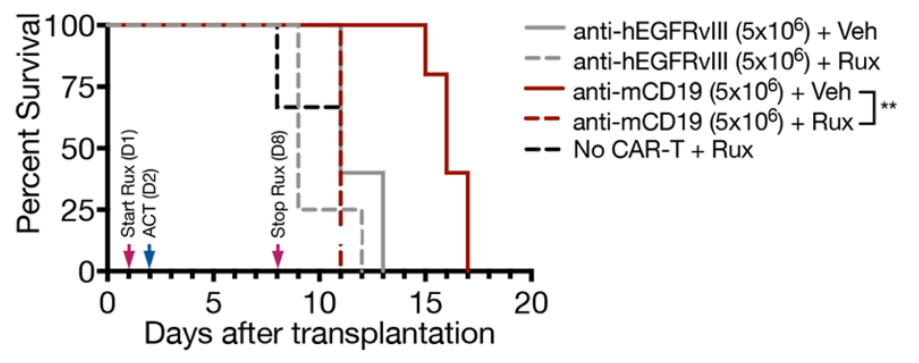


bioRxiv preprint doi: https://doi.org/10.1101/2022.02.15.480217; this version posted February 17, 2022. The copyright holder for this preprint (which was not certified by peer review) is the author/funder. All rights reserved. No reuse allowed without permission.

\section{Ramos, Koch, and Liu et al: Confidential}

772 Figure 4

a

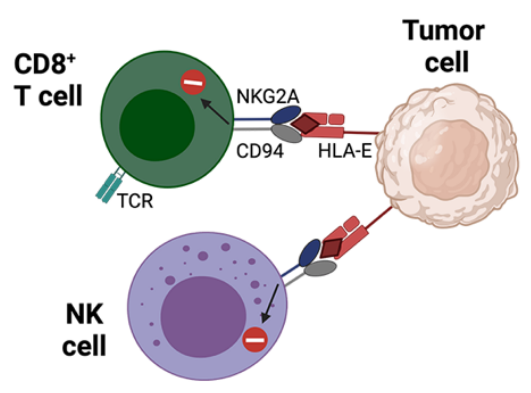

b

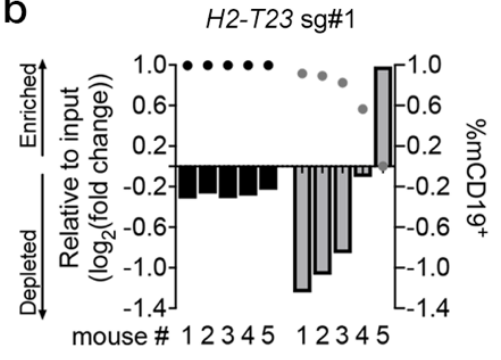

- $\alpha$-hEGFRvIII CAR-T aa-mCD19 CAR-T
C $\quad H 2-T 23 \mathrm{sg} \# 2$

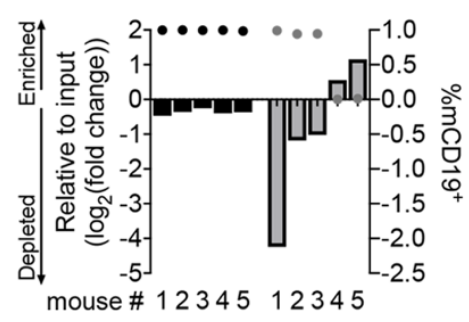

$\bullet \% m C D 19^{+}$B-ALL cells
C

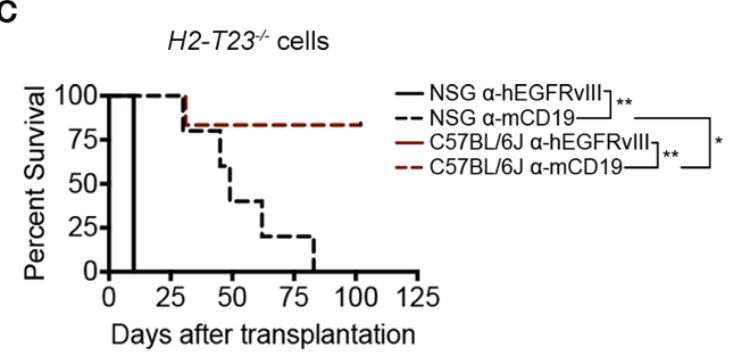

d

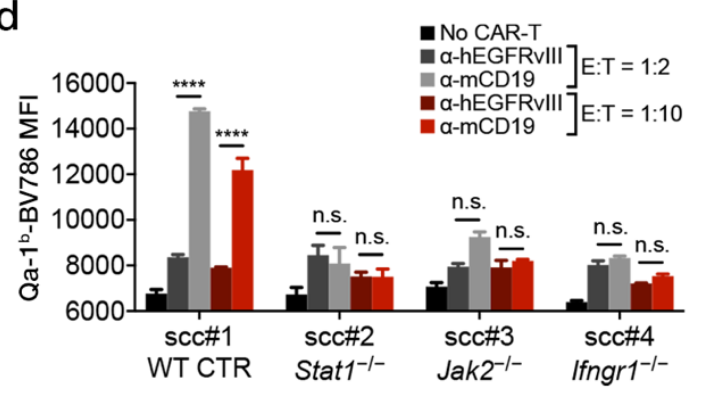


bioRxiv preprint doi: https://doi.org/10.1101/2022.02.15.480217; this version posted February 17,2022 . The copyright holder for this preprint (which was not certified by peer review) is the author/funder. All rights reserved. No reuse allowed without permission.

Ramos, Koch, and Liu et al: Confidential

Figure 5

a

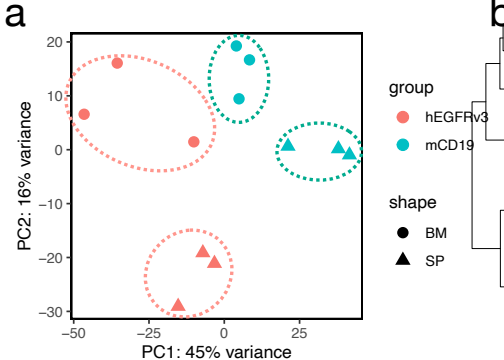

C

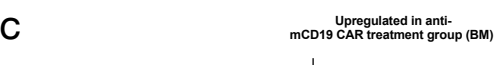

HALLMARK_INTERFERON_ALPHA_RESPONSE. HALLMARK_INTERFERON_GAMMA_RESPONSE HALLMARK_ALLOGRAFT_REJECTION HALLMARK_MYC_TARGETS_V2 HALLMARK_IL6_JAK_STAT3_SIGNALIN

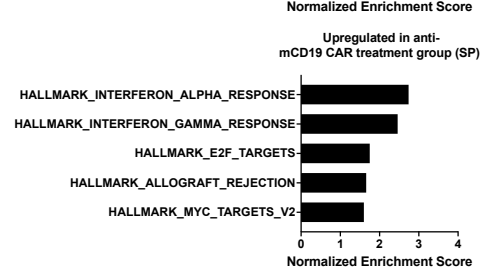

e

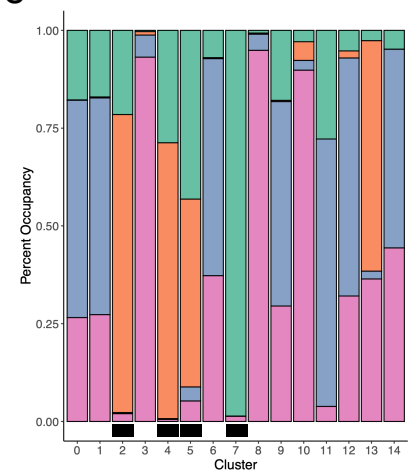

f

Cluster 2 IFNgamma Response / IFNalpha Response / Allograft Rejection

Cluster 4 OXPHOS / E2F Targets / DNA Repair / MYC Targets v1 / mTORC1 Signaling

Cluster 5 OXPHOS / E2F Targets / MYC Targets v1 / MTORC1 Signaling

Cluster 7 mTORC1 Signaling / MYC Targets v1 / E2F Targets / Unfolded Protein Response / G2M Checkpoint

h
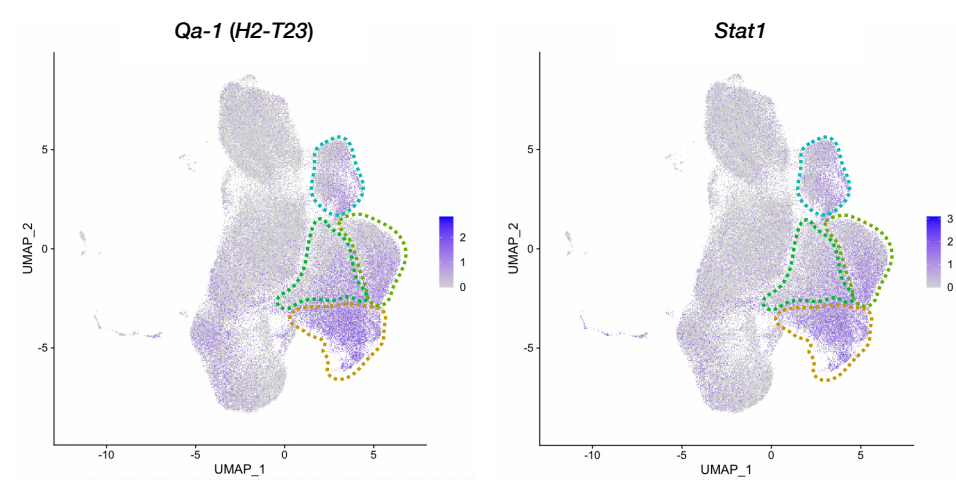

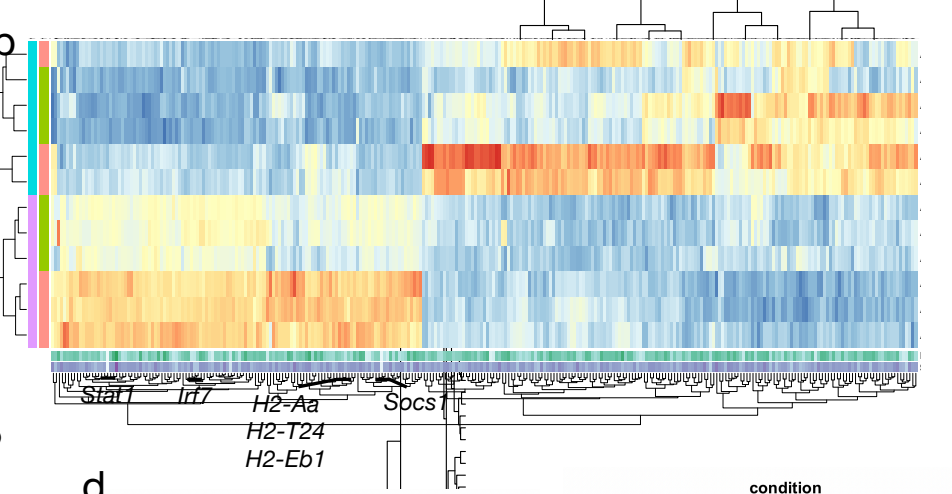
2 treatment
$\begin{array}{lll}1.5 & \text { hEGFRv3 } \\ \text { mCD19 }\end{array}$
$\begin{array}{lr}1 & \text { tissue } \\ 0.5 \quad \mathrm{BM}\end{array}$
$0 \quad \mathrm{SP}$
-0.5 SP.screen.score
$-1$
$-2.5$

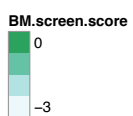

d
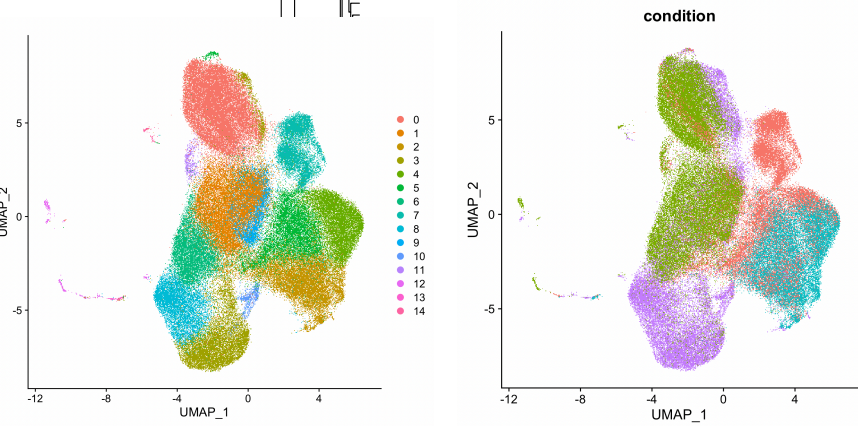

- BM CD19

- BM_V3

- SP_CD19

- SP_V3 g

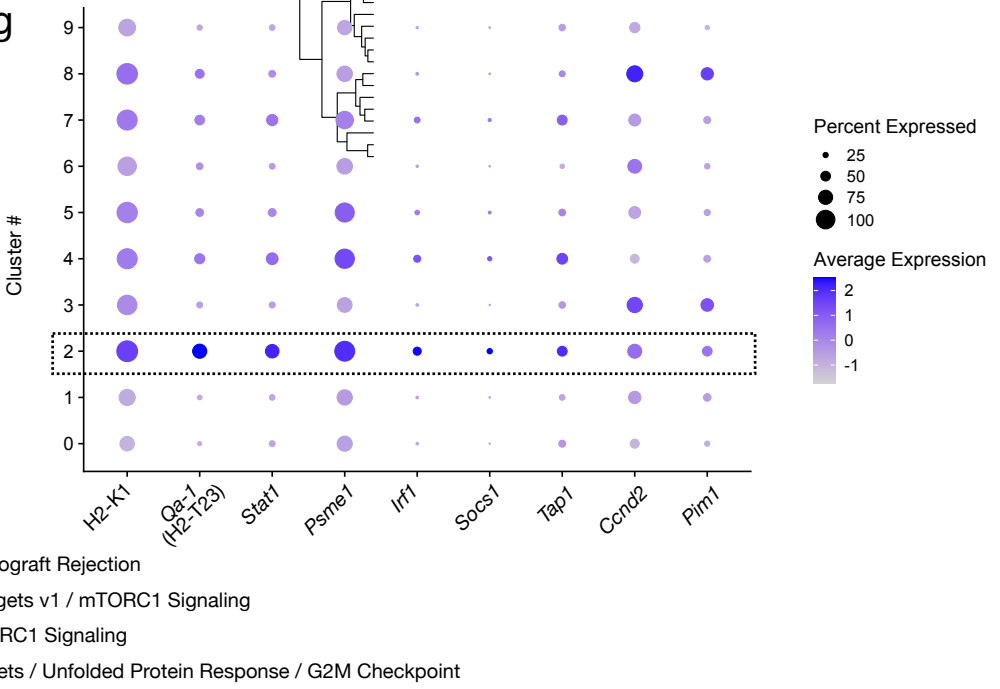


bioRxiv preprint doi: https://doi.org/10.1101/2022.02.15.480217; this version posted February 17, 2022. The copyright holder for this preprint (which was not certified by peer review) is the author/funder. All rights reserved. No reuse allowed without permission.

\section{Ramos, Koch, and Liu et al: Confidential}

796

Figure 6

a

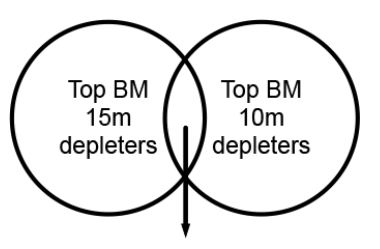

Sensitizer signature

C

ISG.RS signature expression

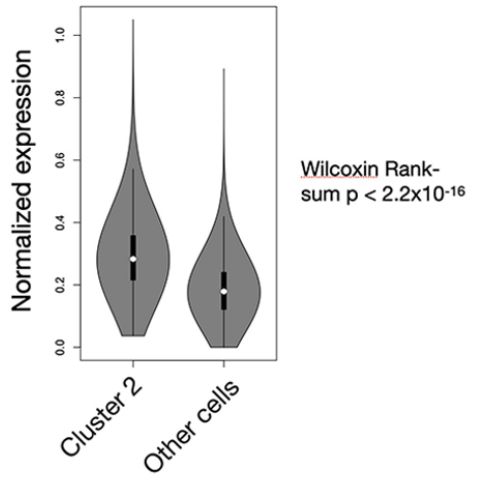

b

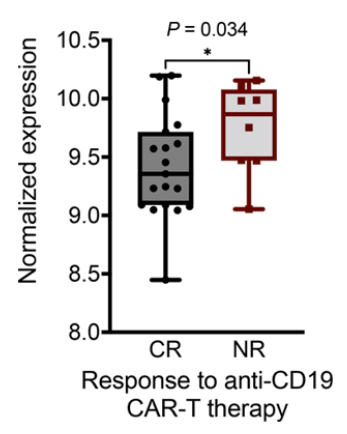

JAKISTAT/MHC-I signature

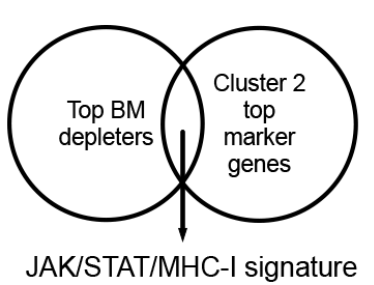

JAKISTAT/MHC-I signature

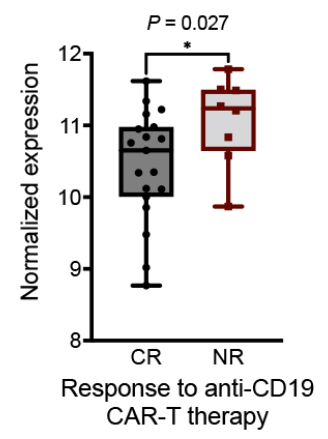

d

IFNYO $\circ^{\circ}$

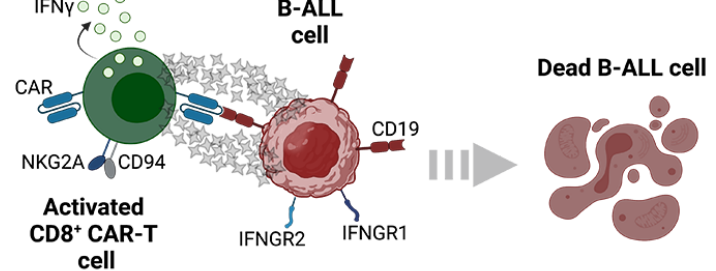

Dead B-ALL cell
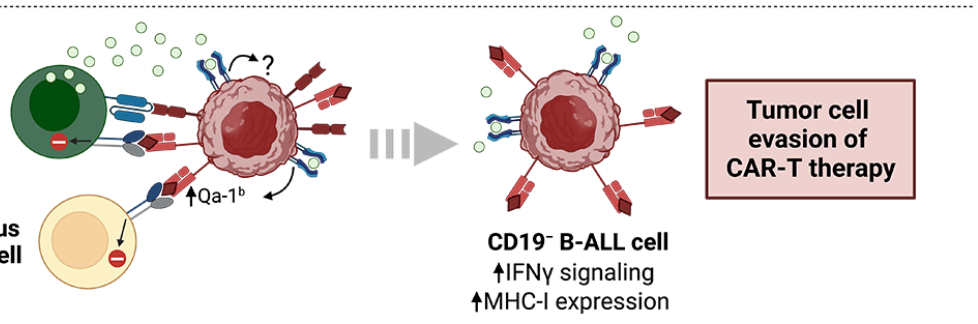


\section{Supplementary Figure 1}

a

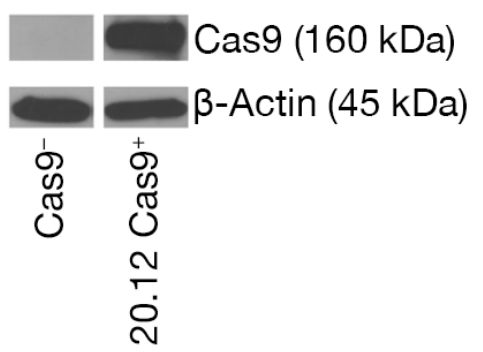

C

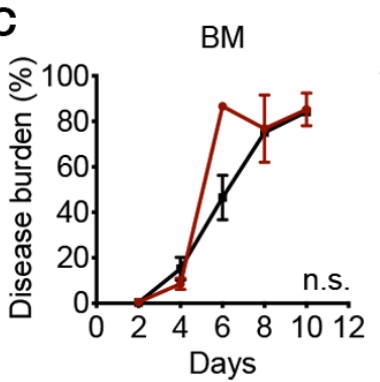

d
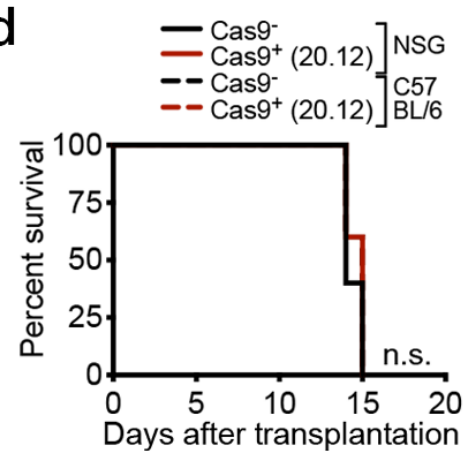

f
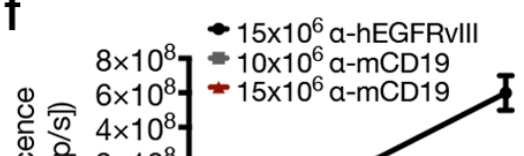

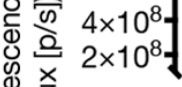

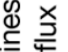

흘 뜽

$8 \times 10^{7}$

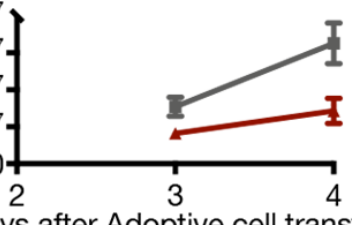

Days after Adoptive cell transfer

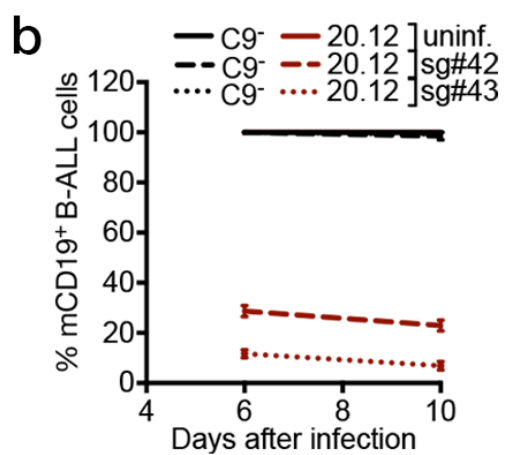

Days after infection
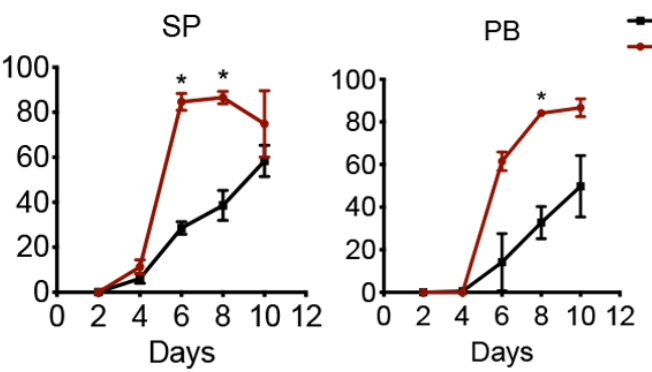

e
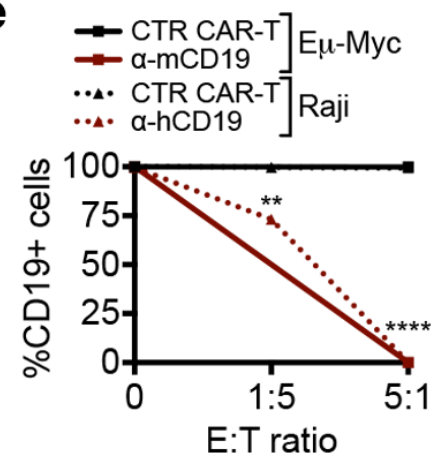

9

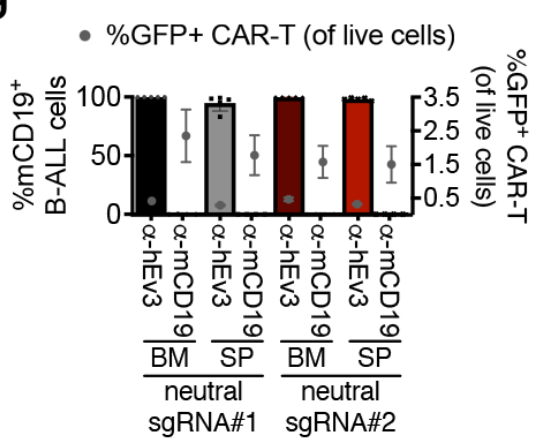

h

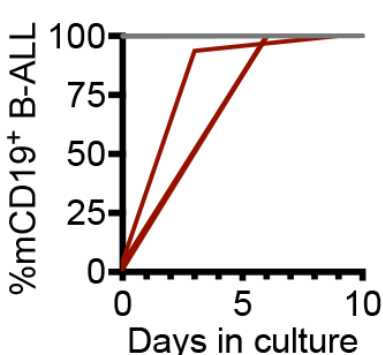

i $\frac{0}{\bar{d}}$

814

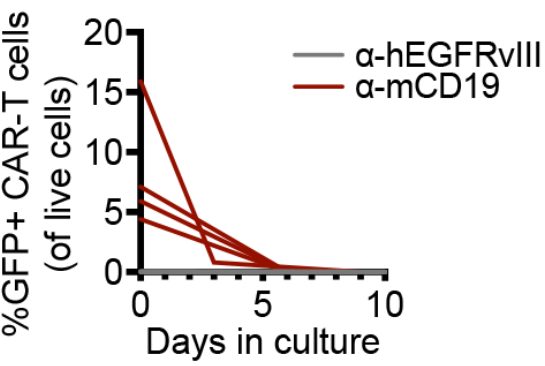


bioRxiv preprint doi: https://doi.org/10.1101/2022.02.15.480217; this version posted February 17, 2022. The copyright holder for this preprint (which was not certified by peer review) is the author/funder. All rights reserved. No reuse allowed without permission.

Ramos, Koch, and Liu et al: Confidential

Supplementary Figure 2

a

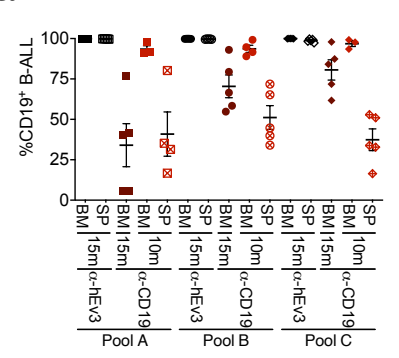

b

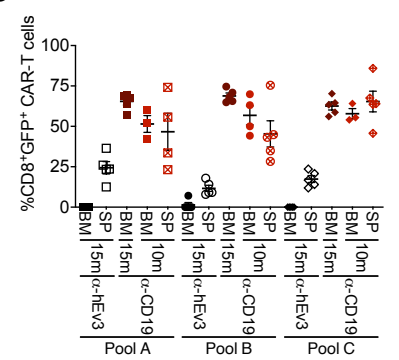

d

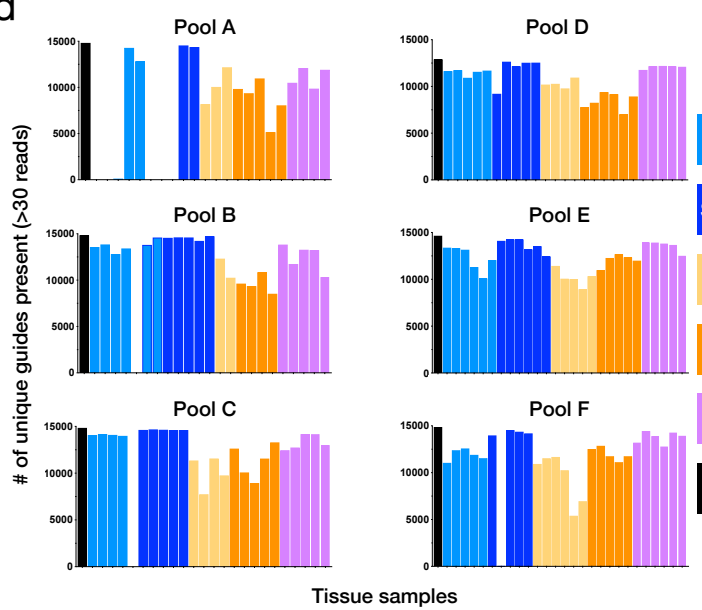

e
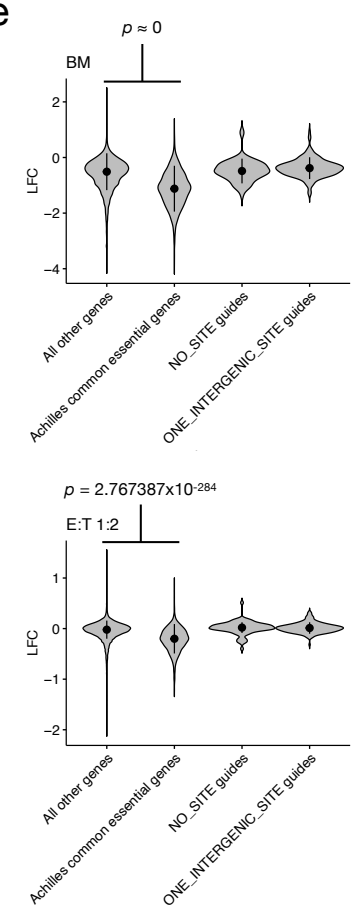
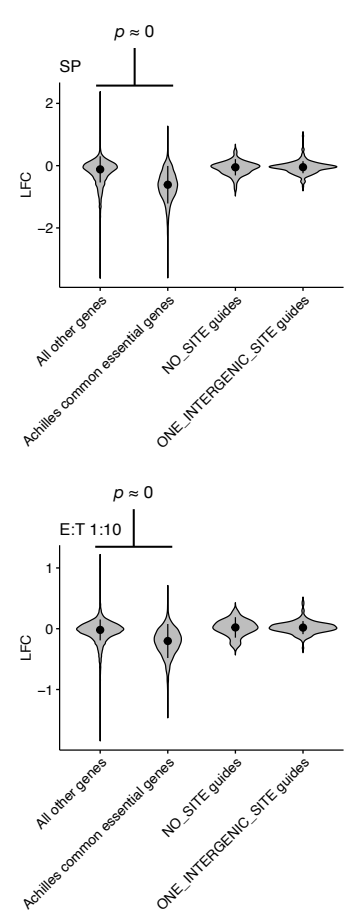

C

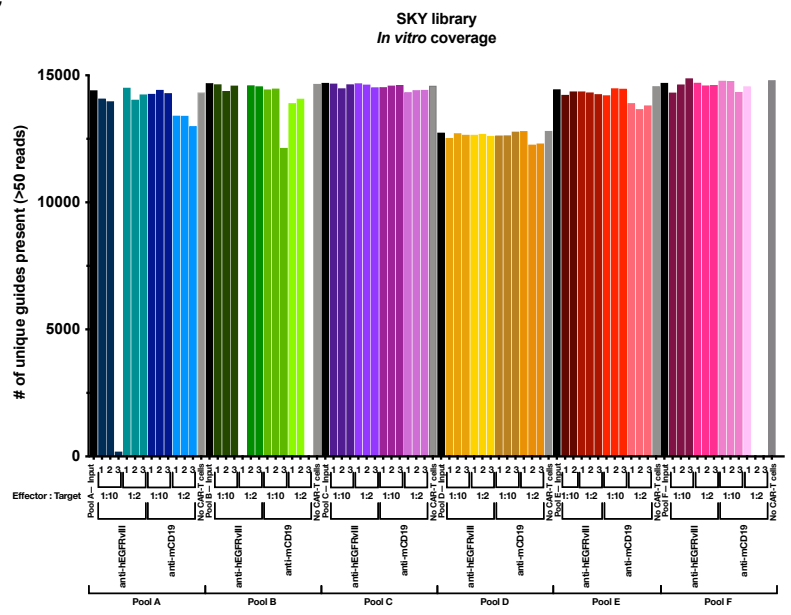

SP hEGFRv3 $15 \mathrm{~m}$

BM mCD19 10m

BM mCD19 15m

SP mCD19 10m

Pool input
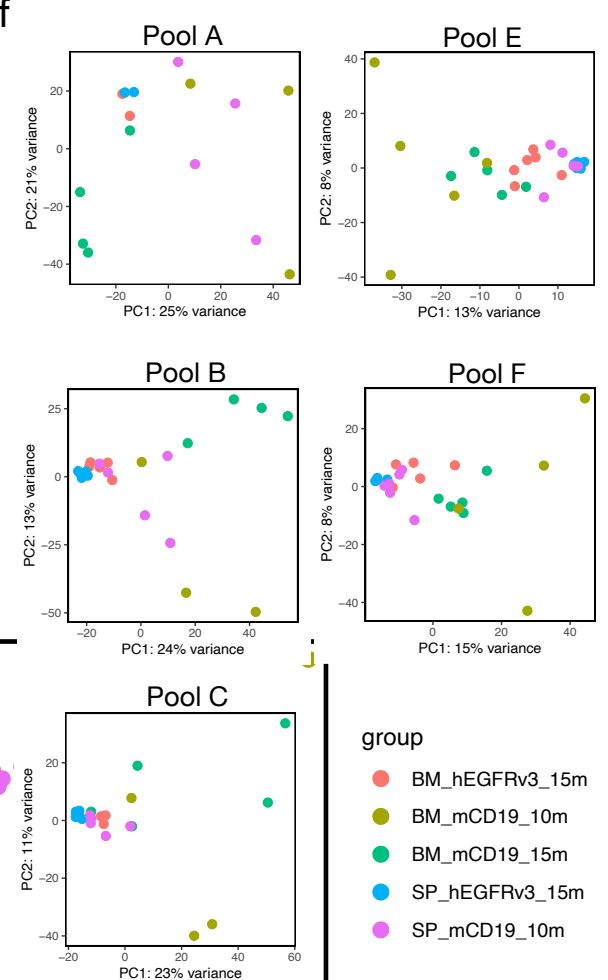

group

- BM_hEGFRv3_15m

- BM_mCD19_10m

- BM_mCD19_15m

- SP_hEGFRv3_15m

- SP_mCD19_10m 
bioRxiv preprint doi: https://doi.org/10.1101/2022.02.15.480217; this version posted February 17, 2022. The copyright holder for this preprint (which was not certified by peer review) is the author/funder. All rights reserved. No reuse allowed without permission.

\section{Ramos, Koch, and Liu et al: Confidential}

\section{Supplementary Figure 3}

a
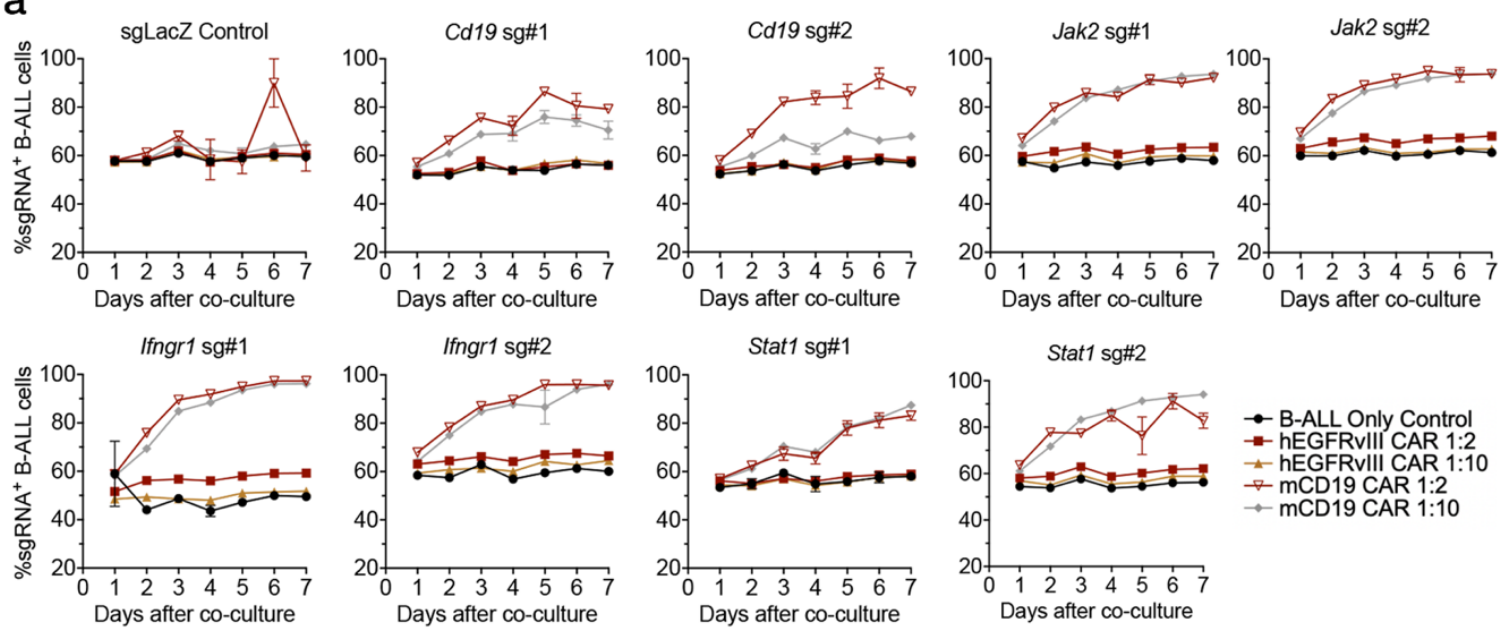

b

C
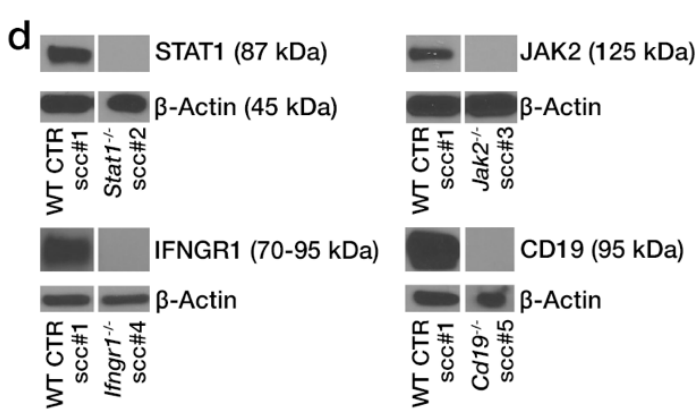

e
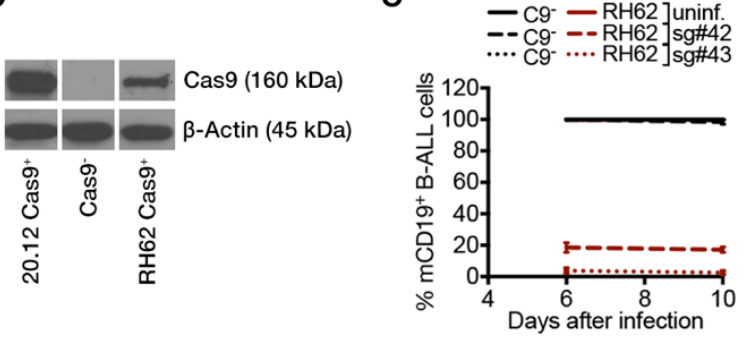

f

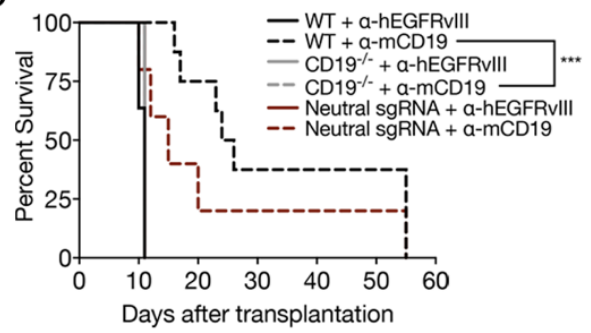

IFNg Blocking Antibody
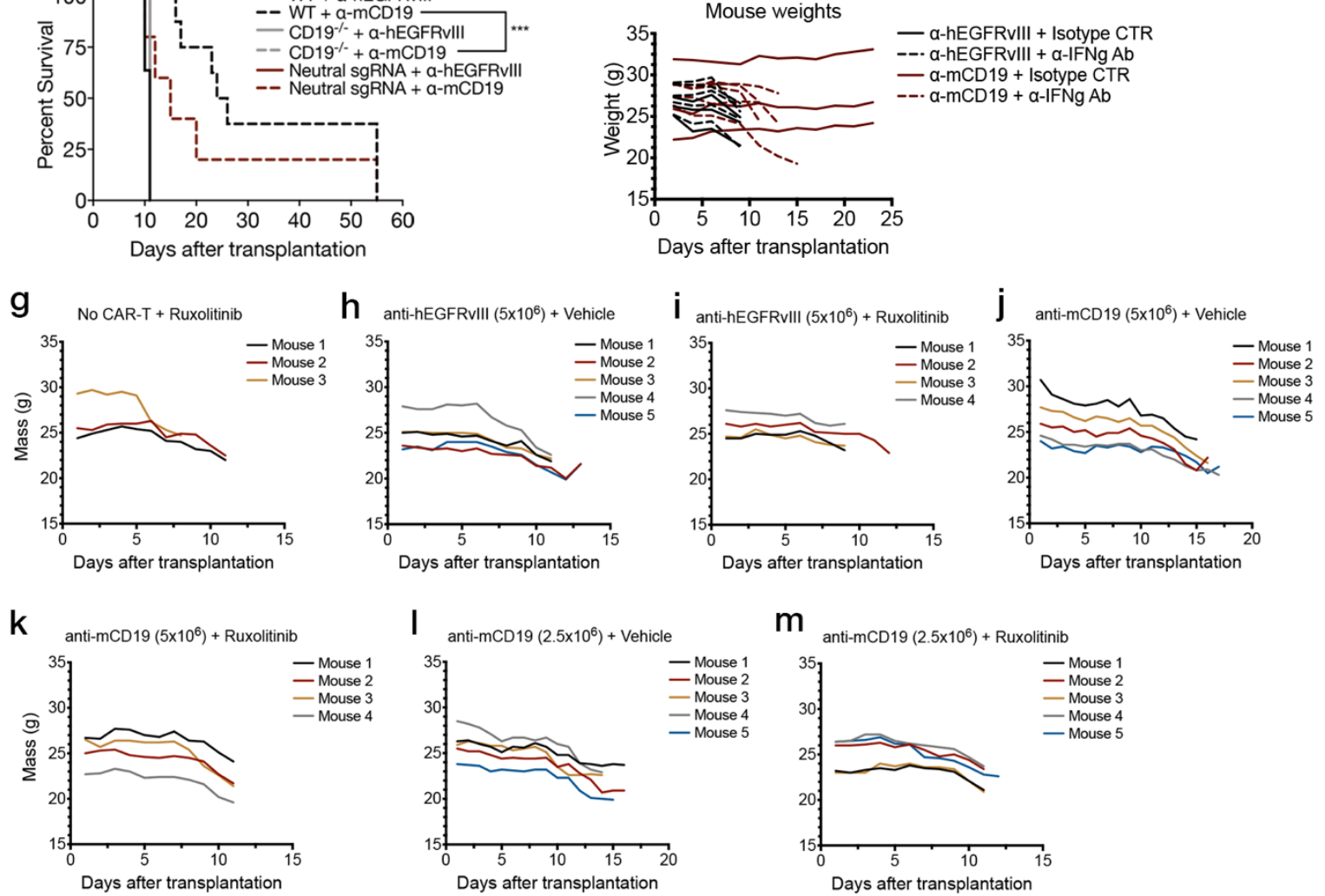

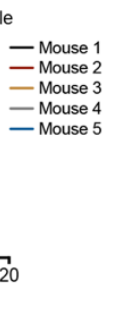

Days after transplantation 
bioRxiv preprint doi: https://doi.org/10.1101/2022.02.15.480217; this version posted February 17,2022 . The copyright holder for this preprint (which was not certified by peer review) is the author/funder. All rights reserved. No reuse allowed without permission.

\section{Ramos, Koch, and Liu et al: Confidential}

\section{Supplementary Figure 4}

a

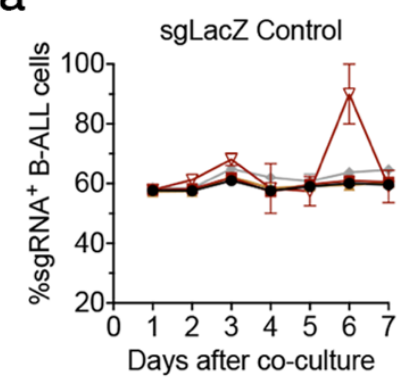

H2-T23 sg\#1

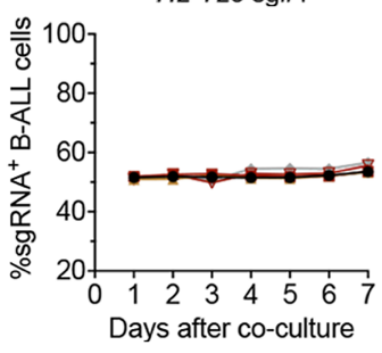

d

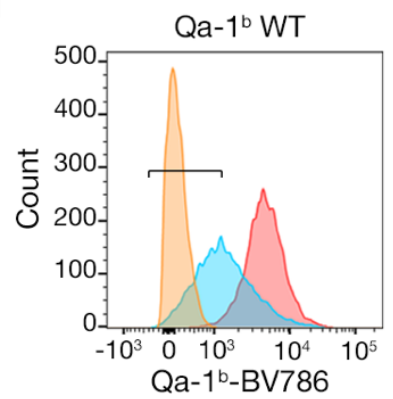

$\square$ Isotype control $\square-$ IFNg stimulation
Cd19 sg\#1

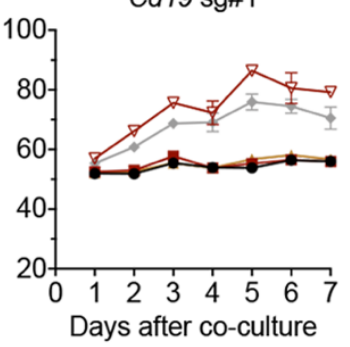

H2-T23 sg\#2

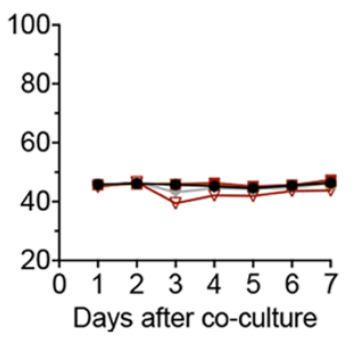

e

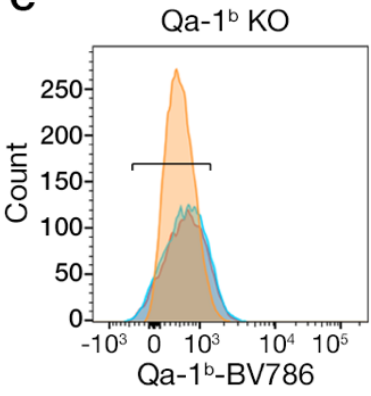

b

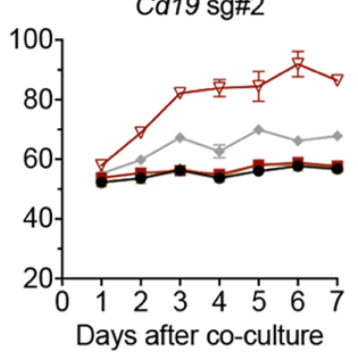

C

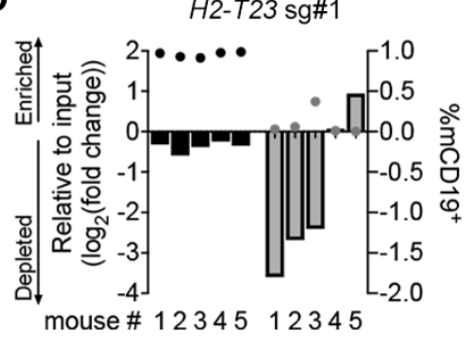

C

H2-T23 sg\#2
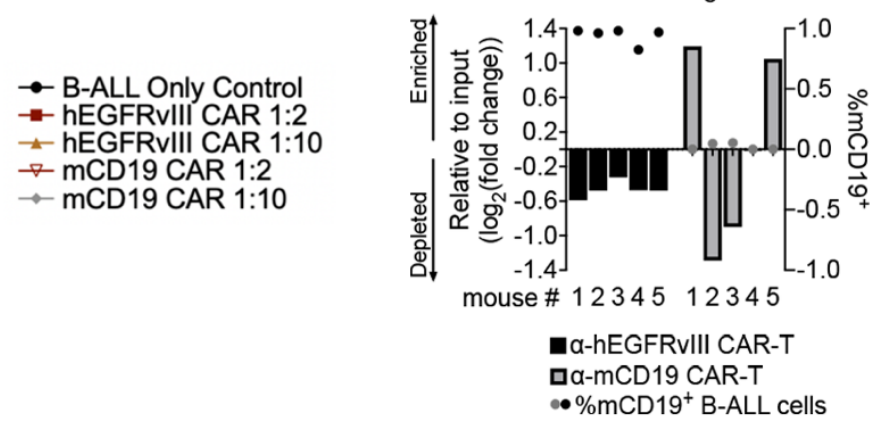

f

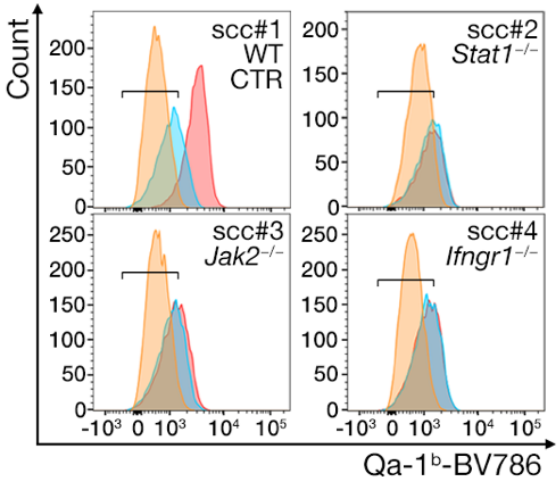

Qa-15-BV786

a-mCD19 CAR-T

$\bullet \% \mathrm{mCD} 19^{+} \mathrm{B}-\mathrm{ALL}$ cells 
bioRxiv preprint doi: https://doi.org/10.1101/2022.02.15.480217; this version posted February 17,2022 . The copyright holder for this preprint (which was not certified by peer review) is the author/funder. All rights reserved. No reuse allowed without permission.

\section{Ramos, Koch, and Liu et al: Confidential}

\section{Supplementary Figure 5}

a

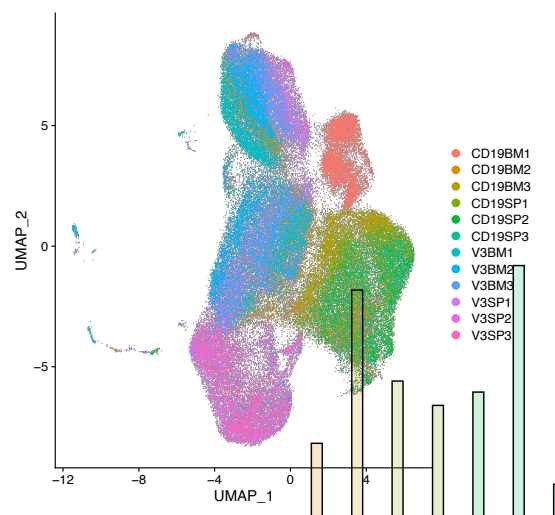

C

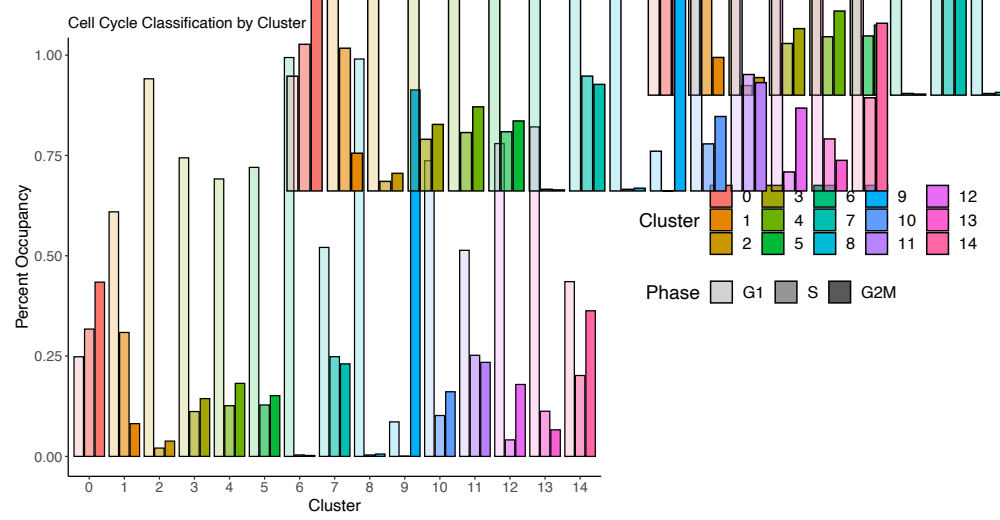

d

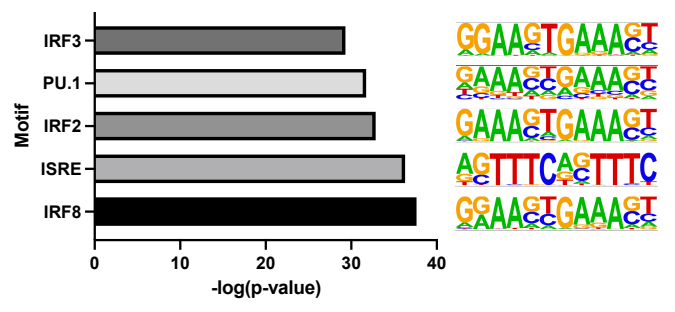

Cluster 5

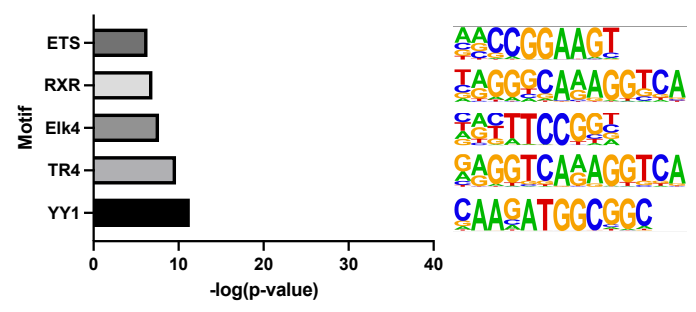

Cluster 4

Cluster 7 b
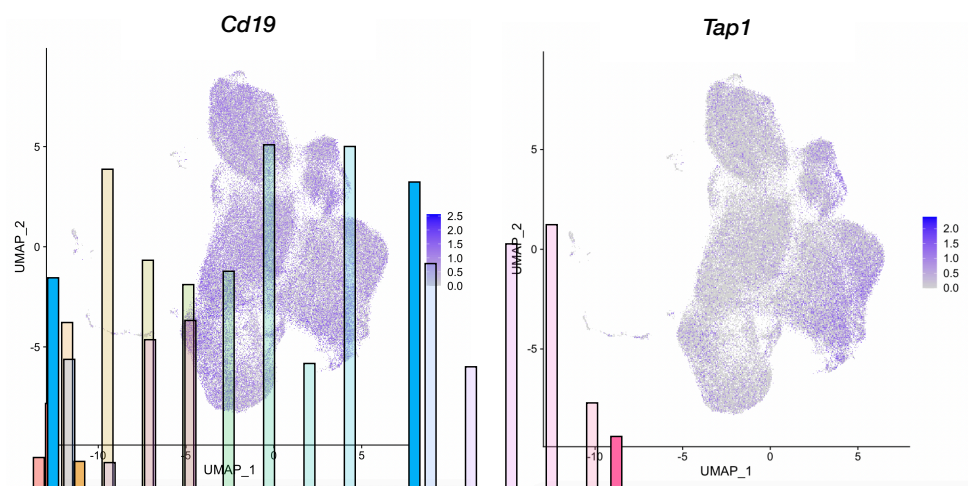
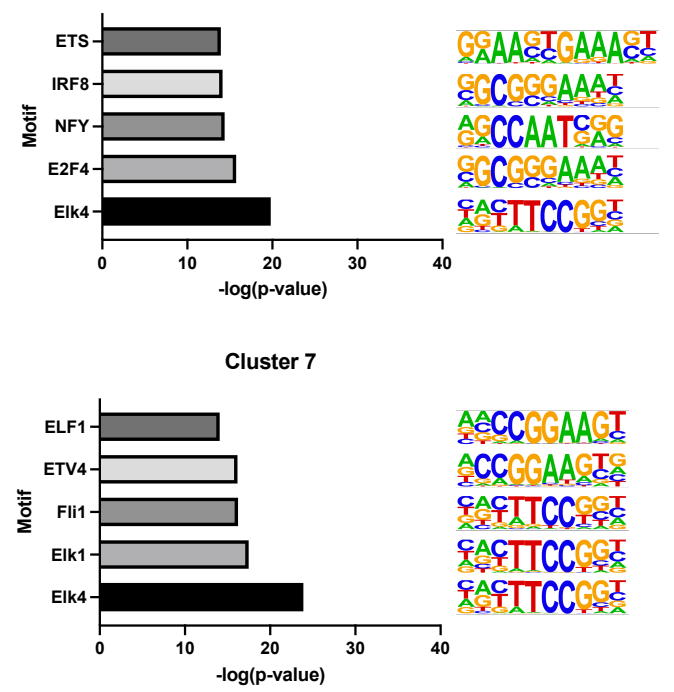


\section{Figure Legends}

840 Figure 1. A fully immunocompetent mouse model of BCR-ABL1+ ${ }^{+}$B-ALL enables parallel in vivo and in vitro genome-wide screens for CAR-T resistance. (a) Survival curves and analysis 842 of irradiated B6 mice inoculated with syngeneic B-ALL cells and treated with indicated CAR-T cell type. Mice treated with CAR-T cells targeting murine CD19 (mCD19) showed significant life

844 extension in a dose-dependent manner, as compared to those treated with no CAR-T cells or control CAR-T cells against human EGFRvIII (hEGFRvIII or hEGFRv3). Bioluminescence imaging

846 four days after adoptive cell transfer (ACT) showed significant disease burden in (b) mice not treated with CAR-T cells and (c) mice treated with control CAR-T cells. Conversely, mice treated

848 with mCD19 CAR-T cells at a dose of either (d) $7 \times 10^{6}$ or (e) $10^{7}$ show (f) significant disease suppression. (g) In vitro cytotoxicity assays show significant depletion of tumor cells along with

850 (h) massive target epitope loss when B-ALL cells are treated with anti-mCD19 CAR-T cells at increasing effector to target cell (E:T) ratios. (i) Concomitantly, CAR-T cells expand and (j) release

852 IFN $\gamma$ when co-cultured with mCD19+ B-ALL cells. $(k-m)$ Experiments to determine the appropriate dose of CAR-T cells for either the marrow (BM) or spleen (SP). Peripheral blood (PB) was also

854 assessed. Mice sacrificed four days after ACT showed an $80 \%$ reduction in marrow disease at a dose of $1.5 \times 10^{7}$ anti-mCD19 CAR-T cells, while identical disease suppression in the spleen and blood was accomplished with $10^{7}$ CAR-T cells. (I) Massive target epitope loss and $(\mathrm{m})$ significant CAR-T cell persistence was also observed in all of the organs harvested from mice treated with anti-mCD19 CAR-T cells. ( $n$ ) Schematic showing the overall design and (o) lentiviral backbone used to create the SKY library. ( $p$ ) Retroviral vectors encoding the 1D3 single chain variable

860 fragment (scFv) targeting mCD19 (top) and the 3C10 scFv targeting hEGFRvlll (bottom). (q) Diagram of screening layout. Significance for survival experiments was determined using log-rank

862 tests. For all other experiments, significance is determined using unpaired two-sided student's ttests with Bonferroni correction for multiple comparisons, or using one-way ANOVA with Turkey's

864 correction for multiple comparisons when more than two groups were compared. Data are mean \pm s.e.m.; $n=4-6$ mice per group). ${ }^{*} \mathrm{P}<0.05 ;{ }^{* *} \mathrm{P}<0.01 ;{ }^{* * *} \mathrm{P}<0.001$; ${ }^{* * *} \mathrm{P}<0.001$.

Figure 2. In vivo genome-wide CRISPR-Cas9-mediated knockout screen identifies Jak/Stat 868 and antigen presentation pathways involved in resistance to CAR-T therapy. (a) Schematic of the data analysis and screen hit discovery workflow. (b-c) Waterfall plots of log-fold changes of

870 the representation of gRNA against different genes in anti-mCD19 CAR-T cell treated animals (b) or cells (c) compared to anti-hEGFRvIlI CAR-T cell treated (control) animals (b) or cells (c). Genes

872 are ranked by the average log-fold changes of all gRNAs against each gene, and point sizes are 
proportional to the magnitude (absolute value) of log-fold changes. Top 5 genes with 874 enriching/depleting gRNAs are labeled. (d) Waterfall plots of the relative significance of each hit (vertical axis, showing log2(fold change) from two-sided student's t-test with correction for multiple 876 comparisons or STARS analysis) versus guide ranks. (e) Relative enrichment/depletion of individual guides against top depleters Ifngr1, Jak2, Stat1, and Qa-1 are shown in each arm of

878 the screen. Guide RNAs against Cd19 are also shown as an indicator of CAR-T treatment pressure. (f) Venn diagrams showing overlap of top hits from the in vitro and in vivo arms of the 880 screen. Top hits are defined as genes with an absolute log-fold change greater than 2. (g) Bar plots of GSEA analysis of top depleting (black) or enriching (orange) hits in each arm of the

882 screen. Plots show up to the top 5 Hallmark pathways with enrichment FDR value smaller than 0.05 enriched Hallmark pathways. (h) Enrichment of KEGG terms within genes showing top gRNA

884 depletion in the in vivo arms of the screen. Shown is a heatmap of participation of the top genes in each enriched pathway.

Figure 3. Loss of components of the IFNY/JAK/STAT pathway sensitizes tumors to CAR-T

888 therapy in vivo. (a) Schematic showing the IFNY signaling pathway. (b-c) In vivo competitive assays demonstrate specific log-fold depletion of Cas9 ${ }^{+}$RH62 B-ALL cells lacking components 890 of the IFNy/JAK/STAT pathway and enrichment of B-ALL cells lacking mCD19 after treatment with anti-mCD19 CAR-T cells in both (b) the bone marrow and (c) the spleen. (d)

892 Immunocompetent (B6) and (e) immunocompromised (NSG) mice transplanted with B-ALL cells deficient in indicated components of the IFNY/JAK/STAT pathway survive significantly longer than

894 control mice. (f) Layout and treatment schedule for anti-IFNy blocking antibody experiment. (g) Blocking IFNy signaling concurrently with CAR-T therapy eliminates the antitumor capabilities of

896 CAR-T cells. Concurrent treatment of Ruxolitinib, a JAK1/2 inhibitor, with either $2.5 \times 10^{6}(\mathrm{~h})$ or $5 \times 10^{6}$ (i) CAR-T cells also eliminates any benefit of CAR-T therapy. Significance for survival 898 experiments was determined using log-rank tests. For all other experiments, significance is determined using unpaired two-sided student's t-tests with Bonferroni correction for multiple 900 comparisons. Data are mean \pm s.e.m.; $n=5-8$ mice per group). ${ }^{*} \mathrm{P}<0.05 ;{ }^{* *} \mathrm{P}<0.01 ;{ }^{* *} \mathrm{P}<0.001$; ${ }^{* * *} \mathrm{P}<0.001$.

902

Figure 4. Loss of Qa-1 ${ }^{b}$, a component of the MHC-I pathway, sensitizes B-ALL cells to CAR-

904 T therapy in vivo. (a) Schematic showing the known effect of the HLA-E/NKG2A/CD94 axis in $\mathrm{NK}$ and $\mathrm{CD}^{+} \mathrm{T}$ cells. (b, c) In vivo competitive assays using two independent guides against the non-classical MHC-I gene H2-T23. Tumors harvested from the bone marrow of relapsed mice 
demonstrate significant depletion of H2-T23 null populations after treatment with anti-mCD19 908 CAR-T cells only when H2-T23 null cells also relapse mCD19+. (c) Immunocompetent (B6) mice transplanted with $\mathrm{Qa}-1^{\mathrm{b}}$-deficient B-ALL cells show increased survival as compared to

910 immunocompromised (NSG) after treatment with anti-mCD19 CAR-T cells. (d) Mean fluorescence intensity (MFI) of Qa-1 ${ }^{\mathrm{b}}$-Brilliant Violet 786 (BV786) after in vitro CAR-T treatment of single cell

912 clones (Scc) deficient in indicated components of the IFNy/JAK/STAT pathway at two different $\mathrm{E}: \mathrm{T}$ ratios, as assayed using flow cytometry. Wildtype control single cell clones were also

914 generated and assayed at the same time. Representative data for WT control clones is shown in (d). Significance for survival experiments was determined using log-rank tests. For all other 916 experiments, significance is determined using unpaired two-sided student's t-tests with Bonferroni correction for multiple comparisons. Data are mean \pm s.e.m.; $n=5-8$ mice per group). * $\mathrm{P}<0.05$;

$918 \quad{ }^{* *} \mathrm{P}<0.01 ;{ }^{* *} \mathrm{P}<0.001 ;{ }^{* * *} \mathrm{P}<0.001$.

920 Figure 5. Bulk and single cell gene expression profiling pinpoints specific cell subsets and expression programs as a source of CAR-T cell resistance in B-ALL. (a) PCA plot of bulk

922 RNA-seq profiles of bone marrow (BM) and spleen (SP) samples collected from mice treated with either anti-mCD19 or anti-hEGFRvIlI (control) CAR T cells. (b) Heatmap of genes differentially 924 expressed between anti-mCD19 and anti-hEGFRvIll CAR T cell-treated samples. Scores of genes from the screen are shown next to each gene's expression profile (column). Top depleters

926 in the bone marrow and spleen samples are labeled. (c) Top Hallmark gene sets enriched among genes overexpressed in mCD19 CAR T cell therapy compared to control treated bone marrow

928 (BM) or spleen (SP) samples. Bar graphs show normalized enrichment scores for each of the top 5 enriched pathways. Enrichment analysis was performed using GSEA and all pathways shown

930 have FDR < 0.05. (d) 2-dimensional UMAP plots of single cell gene expression profiles collected from mice treated with either anti-mCD19 or anti-hEGFRvIII CAR T cell therapy. Data are

932 collected from $n=3$ mice per treatment group. Left panel: cells color-coded by clusters discovered through unsupervised clustering; right panel: cells color-coded by treatment and tissue groups.

934 (e) Participation of cells from each tissue/treatment group in each cluster. Bar graphs show percentage of cells in each cluster belonging to each tissue/treatment group. Clusters where there

936 is substantial enrichment of cells under anti-mCD19 CAR T therapy are marked with a black box underneath the bar plots. (f) Hallmark gene sets enriched in the 4 treatment-specific clusters as

938 labeled in (e). (g) Dot plot showing relative expression patterns of cluster 2 marker genes which are also among top depleters (log2 fold change < -1.75) in the BM samples of the in vivo screen.

940 Dot size is proportional to the percentage of cells in each cluster expressing each gene and dot 
color indicates average expression of each gene in each cluster. (h) UMAP plots showing expression of Qa-1 and Stat1 in single cell samples, with cells color-coded by expression levels.

944 Figure 6. JAK/STAT signaling is a potential therapeutic target in human B-ALL that can be exploited to enhance CAR-T therapy. (a) A gene signature composed of top depleters in

946 the bone marrow is expressed at a lower level in complete responders (CR) to CAR-T therapy and is thus associated with better outcomes in B-ALL patients. Non-responders (NR) show

948 increased expression of these genes, indicating an association with CAR-T resistance. (b) Higher expression of a JAK/STAT/MHC-I resistance signature is associated with poor outcomes

950 in CAR-T treated patients. (c) After CAR-T failure, relapsed B-ALL cells exhibit increased expression of ICB resistance-associated interferon stimulated genes (ISG.RS) that is also

952 associated with poor outcomes in CAR-T treated large B cell lymphoma patients. (d) A final model for how high JAK/STAT/IFNy signaling in tumor cells can promote resistance to CAR-T

954 therapy. Significance is determined using unpaired two-sided student's t-tests with Bonferroni correction for multiple comparisons. Data are mean \pm s.e.m. . ${ }^{*} P<0.05 ;{ }^{* *} P<0.01 ;{ }^{* * *} P<0.001$; ${ }^{* * *} \mathrm{P}<0.001$.

Supplementary Figure 1. Characterization of Cas9+ disease and target epitope loss after CAR-T treatment. (a) Cas9 expression in 20.12 cells assayed via western blot. (b) In vitro cut assay using two validated, non-overlapping guides against murine CD19 (mCD19) was used to determine Cas 9 cutting efficiency in 20.12 cells. Eight days after the introduction of sgRNAs, more than $75 \%$ of all 20.12 cells are $\mathrm{mCD} 19^{-}$. (c) Tomato ${ }^{+} \mathrm{GFP}^{+}$Cas $9^{+} 20.12$ cells show similar or faster growth kinetics as non-Cas9 expressing parental cells in hematopoietic organs of non-irradiated syngeneic B6 mice, as assayed by flow cytometry. (d) Non-irradiated immunocompetent (B6) mice and immunocompromised (NSG) mice succumbed to both Cas9 ${ }^{-}$and $\mathrm{Cas}^{+}(20.12)$ disease

966 at the same time, indicating that Cas9 is not additionally immunogenic in vivo. (e) Murine CAR-T cells targeting either mCD19 or human CD19 (FMC63 scFv) in vitro induce dose dependent target

968 epitope loss in murine $\mathrm{E} \mu$-Myc and human Raji cells, respectively. (f) Bioluminescence imaging of irradiated B6 mice treated with indicated CAR-T cell type and dose at indicated time after ACT.

970 (g) Organs from moribund control mice that relapsed after treatment with $3.5 \times 10^{6}$ CAR-T cells targeting mCD19 demonstrate ongoing CAR-T engraftment and complete target epitope loss in

972 B-ALL cells. (h) Whole spleens relapsed mice after treatment with $5 \times 10^{6}$ indicated CAR-T cell type were dissociated, cultured in vitro and serially assessed for mCD19+ B-ALL and GFP ${ }^{+}$CAR-

974 T cells. As anti-mCD19 CAR-T cells are depleted out of culture, B-ALL cells re-express mCD19. 


\section{Ramos, Koch, and Liu et al: Confidential}

Significance for survival experiments was determined using log-rank tests. For all other experiments, significance is determined using unpaired two-sided student's t-tests with Bonferroni correction for multiple comparisons. Data are mean \pm s.e.m.; $n=4-8$ mice per group. ${ }^{*}<<0.05$; ${ }^{* *} \mathrm{P}<0.01 ;{ }^{* * *} \mathrm{P}<0.001 ;{ }^{* * * *} \mathrm{P}<0.001$.

980 Supplemental Figure 2. In vitro and in vivo genome-wide CRISPR/Cas9-mediated screens show comprehensive representation of sgRNA libraries and capture differences in sgRNA

982 behavior in between control and anti-mCD19 CAR-T treated mice. (a) Flow cytometry assay investigating the proportion of CD19+ B-ALL cells in the spleen (SP) or bone marrow (BM) after

984 treatment with the indicated CAR-T cell type (anti-CD19 versus control anti-human EGFRvIII) and dose. (b) Flow cytometry assay investigating the proportion of persistent CAR-T cells in the spleen 986 (SP) or bone marrow (BM) after treatment with the indicated CAR-T cell type. Data are mean \pm s.e.m.; $n=3-5$ mice per group. (c) sgRNA library representation in each pooled library in

988 the in vitro screen samples. Each bar shows the number of sgRNAs with more than 30 reads in the sequencing sample for a given pool. (d) Same as (c), but for sgRNA library representation in 990 each pooled library in the in vivo screen samples. (e) Violin plots showing relative depletion/enrichment of control sgRNAs used in the screen. $P$-values are calculated from Wilcoxin rank-sum tests for comparison between pairs of group means. Note that in some cases exact $p$ values cannot be determined due to extremely near-zero values and ties in data and are reported

994 as approximately zero. (f) In vivo screen samples projected onto the first 2 principal components of input-normalized sgRNA count matrix.

Supplemental Figure 3. In vitro cytotoxicity assays in cells deficient for components of the 998 IFNy/JAK/STAT pathway do not capture in vivo sensitization phenotypes to CAR-T therapy. (a) In vitro competitive assays completed in cells transduced with guides against the indicated 1000 IFNY/JAK/STAT pathway or control gene and treated with the indicated CAR-T cell type at two E:T ratios. (b) Western blot showing that Tomato+ RH62 B-ALL cells used for all validation 1002 experiments express Cas9 protein. (c) In vitro cut assay using two validated, non-overlapping guides against murine CD19 (mCD19) was used to determine Cas9 cutting efficiency in RH62 1004 cells. Six days after the introduction of sgRNAs, more than $75 \%$ of all RH62 cells are mCD19-. (d) Western blot analysis of single cell clones (scc) deficient in indicated gene and used in survival 1006 validation experiments. (e) Mice transplanted with parental (WT) or neutral control cells do not significantly differ in their survival after treatment with anti-mCD19 CAR-T cells, while mice 1008 transplanted with $\mathrm{Cd} 19^{-/-}$B-ALL cells show no life extension. (f) Mouse weight monitoring over 
time during co-treatment with CAR-T cells and anti-IFNY blocking antibodies shows no additional toxicity during combination therapy. (g-m) Mouse weight monitoring over time during co-treatment with CAR-T cells and Ruxolitinib shows no additional toxicity during combination therapy. Data

1012 are exact mass in grams over time; $n=3-5$ mice per group. Significance for survival experiments was determined using log-rank tests. Data are mean \pm s.e.m.; $n=5-8$ mice per group. * $P<0.05$; ${ }^{* *} \mathrm{P}<0.01 ;{ }^{* * *} \mathrm{P}<0.001 ;{ }^{* * * *} \mathrm{P}<0.001$.

\section{Supplementary Figure 4. Loss of Qa-1 ${ }^{\mathrm{b}}$ does not sensitize B-ALL cells to CAR-T therapy in} vitro.

1018 a) In vitro competitive assays completed in cells transduced with single guide RNAs (sg) against H2-T23 or the indicated control gene and treated with the indicated CAR-T cell type at two E:T ratios. (b-c) In vivo competitive assays using two independent guides against the non-classical MHC-I gene H2-T23. All mice relapsed with mCD19- splenic disease. (d-e) FACS histograms showing Qa-1 ${ }^{\mathrm{b}}$ expression on cells transduced with a control $\operatorname{sgRNA}\left(\mathrm{Qa}-1^{\mathrm{b}} \mathrm{WT}\right)$ or Qa-1 ${ }^{\mathrm{b}}$ deficient cells after stimulation with IFNy. Black brackets indicate gating for Qa- ${ }^{\mathrm{b}}$-negative cell populations (gating is set to strictly exclude $\mathrm{Qa}-1^{\mathrm{b}}$-positive populations, to ensure pure populations were isolated upon cell sorting). (f) Expression of Qa-1 ${ }^{\mathrm{b}}$-BV786 after in vitro stimulation with IFNy of single cell clones (scc) deficient in indicated components of the IFNY/JAK/STAT pathway, as assayed using flow cytometry. Wildtype control single cell clones were also generated and assayed at the same time. Representative data for WT control clones is also shown in (f). For all flow cytometry experiments, data for a minimum of 10,000 live cells were collected and analyzed. Data are mean \pm s.e.m.; $n=5$ mice per group.

Supplemental Figure 5. Additional characterizations of clusters that emerge from singlecell expression profiling of CAR-T cell treated animals. (a) 2-dimensional UMAP plots of single cell gene expression profiles collected from mice treated with either anti-mCD19 or antihEGFRvIII CAR T cell therapy, with cells color-coded by each individual mouse in the dataset. (b)

1036 UMAP plot showing expression of Cd19 (left panel) and Tap1 (right panel) in the single cell expression dataset. (c) Proportions of cells assigned to each cell cycle stage in each of the 15

1038 clusters identified in the single cell expression data. (d) Transcription factor motif enrichment analysis for signature genes in clusters 2, 4, 5 and 7. Shown are -log10 transformed $p$-values for

1040 top 5 enriched motifs along with their position-weight matrix logos for each cluster. 


\section{References}

1046 1. Tang, J., Shalabi, A. \& Hubbard-Lucey, V. M. Comprehensive analysis of the clinical immuno-oncology landscape. Ann. Oncol. 29, 84-91 (2018).

1048 2. June, C. H. \& Sadelain, M. Chimeric antigen receptor therapy. N. Engl. J. Med. 379, 6473 (2018).

1050 3. June, C. H., O'Connor, R. S., Kawalekar, O. U., Ghassemi, S. \& Milone, M. C. CAR T cell immunotherapy for human cancer. Science (80-. ). 359, 1361-1365 (2018).

1052 4. Locke, F. L. et al. Phase 1 Results of ZUMA-1: A Multicenter Study of KTE-C19 AntiCD19 CAR T Cell Therapy in Refractory Aggressive Lymphoma. Mol. Ther. 25, 285-295 (2017).

5. Maude, S. L. et al. Tisagenlecleucel in children and young adults with B-cell lymphoblastic leukemia. N. Engl. J. Med. 378, 439-448 (2018).

6. Schuster, S. J. et al. Chimeric antigen receptor T Cells in refractory B-Cell lymphomas. $N$. Engl. J. Med. 377, 2545-2554 (2017).

1060

7. Lee, D. W. et al. T cells expressing CD19 chimeric antigen receptors for acute

Lancet 385, 517-528 (2015).

1062 8. Park, J. H. et al. Long-Term Follow-up of CD19 CAR Therapy in Acute Lymphoblastic Leukemia. N. Engl. J. Med. 378, 449-459 (2018).

1064 9. Fry, T. J. et al. CD22-targeted CAR T cells induce remission in B-ALL that is naive or resistant to CD19-targeted CAR immunotherapy. Nat. Med. 24, 20-28 (2018).

1066 10. Hay, K. A. et al. Factors associated with durable EFS in adult B-cell ALL patients achieving MRD-negative CR after CD19 CAR T-cell therapy. Blood 133, 1652-1663

1068 (2019).

11. Schuster, S. J. et al. Tisagenlecleucel in adult relapsed or refractory diffuse large B-cell lymphoma. N. Engl. J. Med. 380, 45-56 (2019).

1072

12. Neelapu, S. S. et al. Axicabtagene ciloleucel CAR T-cell therapy in refractory large B-Cell

13. Wang, M. et al. KTE-X19 CAR T-Cell Therapy in Relapsed or Refractory Mantle-Cell

14. Fraietta, J. A. et al. Determinants of response and resistance to CD19 chimeric antigen

1078 15. Larson, R. C. \& Maus, M. V. Recent advances and discoveries in the mechanisms and functions of CAR T cells. Nat. Rev. Cancer 21, 145-161 (2021).

1080 16. Finney, O. C. et al. CD19 CAR T cell product and disease attributes predict leukemia remission durability. J. Clin. Invest. 129, 2123-2132 (2019).

1082 17. Fraietta, J. A. et al. Determinants of response and resistance to CD19 chimeric antigen receptor (CAR) T cell therapy of chronic lymphocytic leukemia. Nat. Med. 24, 563-571

1084 (2018).

1086

18. Orlando, E. J. et al. Genetic mechanisms of target antigen loss in CAR19 therapy of

19. Grupp, S. A. et al. Chimeric Antigen Receptor-Modified T Cells for Acute Lymphoid

20. Majzner, R. G. \& Mackall, C. L. Tumor antigen escape from car t-cell therapy. Cancer

$1090 \quad$ Discov. 8, 1219-26 (2018).

$1092 \quad$ Lymphoblastic Leukemia and Its Prevention and Treatment Strategies. Front. Immunol. 10, 1-15 (2019).

1094 22. Singh, N. et al. Impaired Death Receptor Signaling in Leukemia Causes Antigen- 
Independent Resistance by Inducing CAR T-cell Dysfunction. Cancer Discov. 10, 552567 (2020).

23. Williams, R. T., Roussel, M. F. \& Sherr, C. J. Arf gene loss enhances oncogenicity and limits imatinib response in mouse models of Bcr-Abl-induced acute lymphoblastic leukemia. Proc. Natl. Acad. Sci. U. S. A. 103, 6688-6693 (2006).

1100 24. Williams, R. T., Besten, W. Den \& Sherr, C. J. Cytokine-dependent imatinib resistance in mouse. Genes Dev. 21, 2283-2287 (2007).

1102 25. Meacham, C. E. et al. A genome-scale in vivo loss-of-function screen identifies phf6 as a lineage-specific regulator of leukemia cell growth. Genes Dev. 29, 483-488 (2015).

1104 26. Fiedler, E. R. C., Bhutkar, A., Lawler, E., Besada, R. \& Hemann, M. T. In vivo RNAi screening identifies Pafah1b3 as a target for combination therapy with TKIs in BCRABL11 BCP-ALL. Blood Adv. 2, 1229-1242 (2018).

1108

27. Doench, J. G. et al. Rational design of highly active sgRNAs for CRISPR-Cas9-mediated gene inactivation. Nat. Biotechnol. 32, 1262-1267 (2014).

28. Kochenderfer, J. N., Yu, Z., Frasheri, D., Restifo, N. P. \& Rosenberg, S. A. Adoptive transfer of syngeneic $T$ cells transduced with a chimeric antigen receptor that recognizes murine CD19 can eradicate lymphoma and normal B cells. Blood 116, 3875-3886 (2010). Davila, M. L., Kloss, C. C., Gunset, G. \& Sadelain, M. CD19 CAR-Targeted T Cells Induce Long-Term Remission and B Cell Aplasia in an Immunocompetent Mouse Model of B Cell Acute Lymphoblastic Leukemia. PLoS One 8, 1-14 (2013).

30. Paszkiewicz, P. J. et al. Targeted antibody-mediated depletion of murine CD19 CAR T cells permanently reverses B cell aplasia. J. Clin. Invest. 126, 4262-4272 (2016).

31. Boulch, M. et al. A cross-talk between CAR T cell subsets and the tumor

1120 32. Sotillo, E. et al. Convergence of acquired mutations and alternative splicing of CD19 enables resistance to CART-19 immunotherapy. Cancer Discov. 5, 1282-1295 (2015).

1122 33. Dufva, O. et al. Integrated drug profiling and CRISPR screening identify essential pathways for CAR T-cell cytotoxicity. Blood 135, 597-609 (2020).

1124 34. Creelan, B. C. \& Antonia, S. J. The NKG2A immune checkpoint - a new direction in cancer immunotherapy. Nat. Rev. Clin. Oncol. 16, 277-278 (2019).

1126 35. Borst, L., van der Burg, S. H. \& van Hall, T. The NKG2A-HLA-E axis as a novel checkpoint in the tumor microenvironment. Clin. Cancer Res. 26, 5549-5556 (2021).

1128 36. Braud, V. et al. HLA-E binds to natural killer cell receptors CD94/NKG2A, B and C. Nature 391, 795-799 (1998).

1130 37. Braud, V. M., Aldemir, H., Breart, B. \& Ferlin, W. G. Expression of CD94-NKG2A inhibitory receptor is restricted to a subset of CD8+ T cells. Trends Immunol. 24, 162-164 1132 (2003).

38. Malmberg, K.-J. et al. IFN-y protects short-term ovarian carcinoma cell lines from CTL lysis via a CD94/NKG2A-dependent mechanism. J. Clin. Invest. 110, 1515-1523 (2002).

39. Le Dréan, E. et al. Inhibition of antigen-induced T cell response and antibody-induced NK cell cytotoxicity by NKG2A: Association of NKG2A with SHP-1 and SHP-2 proteintyrosine phosphatases. Eur. J. Immunol. 28, 264-276 (1998).

1138 40. Anfossi, N. et al. Human NK Cell Education by Inhibitory Receptors for MHC Class I. Immunity 25, 331-342 (2006).

1140 41. André, P. et al. Anti-NKG2A mAb Is a Checkpoint Inhibitor that Promotes Anti-tumor Immunity by Unleashing Both T and NK Cells. Cell 175, 1731-1743.e13 (2018).

1142 42. van Montfoort, N. et al. NKG2A Blockade Potentiates CD8 T Cell Immunity Induced by Cancer Vaccines. Cell 175, 1744-1755.e15 (2018).

1144 43. Ruggeri, L. et al. Effects of anti-NKG2A antibody administration on leukemia and normal hematopoietic cells. Haematologica 101, 626-633 (2016). 
1146 44. Jain, M. D. et al. Tumor interferon signaling and suppressive myeloid cells are associated with CAR T-cell failure in large B-cell lymphoma. Blood 137, 2621-2633 (2021).

1148 45. Benci, J. L. et al. Opposing Functions of Interferon Coordinate Adaptive and Innate Immune Responses to Cancer Immune Checkpoint Blockade. Cell 178, 933-948.e14 (2019).

46. Benci, J. L. et al. Tumor Interferon Signaling Regulates a Multigenic Resistance Program to Immune Checkpoint Blockade. Cell 167, 1540-1554.e12 (2016).

47. Restifo, N. P. et al. Loss of Functional Beta2-Microglobulin in Metastatic Melanomas From Five Patients Receiving Immunotherapy. JNCI J. Natl. Cancer Inst. 88, 100-108 (1996).

48. Zaretsky, J. M. et al. Mutations associated with acquired resistance to PD-1 blockade in melanoma. N. Engl. J. Med. 375, 819-829 (2016).

1158 49. Sade-Feldman, M. et al. Resistance to checkpoint blockade therapy through inactivation of antigen presentation. Nat. Commun. 8, (2017).

1160 50. Sucker, A. et al. Acquired IFNy resistance impairs anti-tumor immunity and gives rise to T-cell-resistant melanoma lesions. Nat. Commun. 8, 1-15 (2017).

1162 51. Hart, T. et al. High-Resolution CRISPR Screens Reveal Fitness Genes and GenotypeSpecific Cancer Liabilities. Cell 163, 1515-1526 (2015).

1164 52. Doench, J. G. Am i ready for CRISPR? A user's guide to genetic screens. Nat. Rev. Genet. 19, 67-80 (2018).

1166 53. Patro, R., Duggal, G., Love, M. I., Irizarry, R. A. \& Kingsford, C. Salmon provides fast and bias-aware quantification of transcript expression. Nat. Methods 14, 417-419 (2017).

1168 54. Soneson, C., Love, M. I. \& Robinson, M. D. Differential analyses for RNA-seq: transcriptlevel estimates improve gene-level inferences. F1000Research 4, 1521 (2016).

1170 55. Raghavan, S. et al. Microenvironment drives cell state, plasticity, and drug response in pancreatic cancer. Cell 184, 6119-6137.e26 (2021).

117256 . Tirosh, I. et al. Dissecting the multicellular ecosystem of metastatic melanoma by singlecell RNA-seq. Science (80-. ). 352, 189-196 (2016).

1174 57. Eyquem, J. et al. Targeting a CAR to the TRAC locus with CRISPR/Cas9 enhances tumour rejection. Nature 543, 113-117 (2017).

1176 58. Posey, A. D. et al. Engineered CAR T Cells Targeting the Cancer-Associated TnGlycoform of the Membrane Mucin MUC1 Control Adenocarcinoma. Immunity 44, 14441454 (2016).

59. Engels, B. et al. Retroviral Vectors for High-Level Transgene Expression in T Lymphocytes. Hum. Gene Ther. 14, 1155-1168 (2003).

60. Morgan, R. A. et al. Recognition of glioma stem cells by genetically modified T cells targeting EGFRvIll and development of adoptive cell therapy for glioma. Hum. Gene Ther. 23, 1043-1053 (2012).

1184 61. R Core Team. R: A Language and Environment for Statistical Computing. [online] Vienna, Austria: R Foundation for Statistical Computing. Available at [https://www.R-project.org]. 